\title{
Sobre el calentamiento y la acidificación del océano mundial y su posible expresión en el medio marino costero colombiano
}

\author{
Paula Judith Rojas-Higuera*, José Daniel Pabón-Caicedo
}

Grupo “Tiempo, clima y sociedad”, Departamento de Geografía, Universidad Nacional de Colombia, Bogotá, Colombia

\begin{abstract}
Resumen
Con base en el análisis de datos de la temperatura superficial del mar y del $\mathrm{pH}$ en sectores marinos costeros del mar Caribe y el océano Pacífico colombianos, se identificaron las tendencias de largo plazo que constituyen una expresión regional de los fenómenos globales de calentamiento y acidificación del océano. A partir del establecimiento de estos cambios se infirió su potencial impacto en los ecosistemas marinos de los sectores analizados. Se evidenció un incremento en la temperatura superficial del mar del orden de $0,23{ }^{\circ} \mathrm{C}$ por decenio en el Mar Caribe y de $0,18{ }^{\circ} \mathrm{C}$ por decenio en el océano Pacífico. Se encontró una disminución en el pH de una magnitud de $-0,071$ unidades por decenio y $-0,090$ unidades por decenio para el Caribe y el Pacífico, respectivamente. Se estimó que hacia finales del siglo XXI habría un aumento de la temperatura superficial del mar de hasta $2{ }^{\circ} \mathrm{C}$ en relación con la observada a comienzos de siglo en los sectores analizados del Caribe y el Pacífico. El pH, por su parte, habría disminuido hasta en 0,5 sus valores en las regiones de estudio hacia finales del siglo XXI con el escenario A2, el más drástico de los analizados en este trabajo. Con base en la revisión de las evidencias publicadas por otros autores sobre los impactos causados en los ecosistemas por el reciente aumento de la temperatura del mar y la reducción del pH, es de suponer que los ecosistemas marinos colombianos se verán dramáticamente afectados, toda vez que sufrirán cambios en el ambiente físico mayores que los reportados hasta hoy, una situación que se hará más aguda en la medida que transcurra el siglo XXI.
\end{abstract}

Palabras clave: calentamiento del océano, acidificación del océano, temperatura superficial del mar, $\mathrm{pH}$, impacto de ecosistemas marinos, mar Caribe colombiano, océano Pacífico colombiano.

On the warming and acidification of the global ocean and their possible expression in Colombian marine and coastal environment

\begin{abstract}
Based on the analysis of the data on sea surface temperature and $\mathrm{pH}$ recorded in sectors of the Colombian Caribbean and Pacific coast, we identified the long term trends which locally represent the global ocean warming and acidification phenomena. Based on these changes we deduced the potential impacts on the marine ecosystems. A rise of about $0.23^{\circ} \mathrm{C} /$ decade in the sea surface temperature for the Caribbean Sea and of $0.18^{\circ} \mathrm{C} /$ decade for the Pacific Ocean was evidenced. We also found a reduction of approximately 0,071 units/decade in the $\mathrm{pH}$ for the Caribbean Sea, and of 0,090 units/decade for the Pacific Ocean in the sectors under study. The estimation of the change in the sea surface temperature for the end of the $21^{\text {st }}$ century suggests an increase of about $2^{\circ} \mathrm{C}$ compared to the temperature observed at the beginning of this century. At the end of the $21^{\text {st }}$ century the $\mathrm{pH}$ might be 0,5 units lower under the conditions of the A2 extreme scenario. Based on the review of the evidences published by other authors about the impacts caused on ecosystems by the recent sea temperature increase and the $\mathrm{pH}$ reduction, it can be assumed that Colombian ecosystems will be dramatically impacted given that changes in the physical environment are greater than those reported up today, a situation that will worsen with time.
\end{abstract}

Key words: Ocean warming, ocean acidification, sea surface temperature, $\mathrm{pH}$, marine ecosystems impact, Colombian Caribbean Sea, Colombian Pacific Ocean.

\section{Introducción}

El cambio global que se está presentando en la actualidad y que podría acentuarse en los próximos decenios (Intergovernmental Panel on Climate Change - IPCC, 2013) afectará el ecosistema planetario e impactará sensiblemente los sistemas socioeconómicos de los países del mundo. Diversos autores (IPCC, 2013) han señalado que el ambiente marino, uno de los grandes componentes del ecosistema planetario en donde se desarrollan procesos físicos, químicos, biológicos y ecológicos de los cuales

\footnotetext{
*Corresponding author:

Paula Judith Rojas Higuera, pjrojash@unal.edu.co

Recibido: 12 de octubre de 2014

Aceptado: 13 de mayo de 2015
} 
depende en alto grado el desarrollo de la sociedad mundial, está presentando cambios en sus características físicoquímicas. La incidencia del cambio global en los procesos físicos, químicos y biogeoquímicos de los océanos y costas ha modificado la biodiversidad marina y la estructura ecológica, así como las funciones, beneficios y servicios de los ecosistemas marinos (Fischlin, et al., 2007). Este fenómeno planetario se ha evidenciado en los océanos por el aumento de la temperatura del agua, la acidificación y el aumento del nivel del mar (IPCC, 2013).

Los espacios marinos y costeros son el motor del desarrollo y la base de la supervivencia de las comunidades de diversos países del mundo. En Colombia, cerca de la mitad del territorio está constituida por espacios marinos con una rica variedad de ecosistemas de gran importancia ecológica, socioeconómica y cultural; estos espacios se ven afectados por el cambio global que se presenta en el océano. Los principales efectos de dicho cambio, el calentamiento y la acidificación del agua, así como el ascenso del nivel del mar, constituyen amenazas potenciales para los ecosistemas en las áreas marítimas colombianas. Sin embargo, el grado de amenaza y vulnerabilidad con respecto a los dos primeros son muy poco conocidos en el contexto nacional.

Dada esta situación, es necesario producir conocimiento sobre la expresión regional del calentamiento y la acidificación del océano y su impacto en los ecosistemas marinos y costeros colombianos. Específicamente se debería responder a las siguientes preguntas: ¿Cómo se expresan regionalmente el calentamiento y la acidificación del océano? ¿Cómo afectarían estos fenómenos los ecosistemas marinos y costeros en las áreas marítimas colombianas? ¿Cuáles serían las consecuencias en la sociedad colombiana de este impacto en el medio marino? En este contexto, el propósito del presente estudio fue contribuir a la exploración de las señales de calentamiento y de acidificación del océano y de su impacto en los ecosistemas marinos y costeros en el territorio marítimo colombiano.

\section{El estado del conocimiento en el tema}

El cambio global incide en los procesos físicos, químicos y biogeoquímicos de los océanos y costas, pues modifica su estructura ecológica y sus funciones, así como los beneficios y servicios de los ecosistemas marinos (Fischlin, et al., 2007). Los patrones de distribución de las variables oceánicas (temperatura, salinidad, $\mathrm{pH}$, entre otras) y de circulación marina están asociados al clima predominante en la región. Por ello, el cambio en las variables climatológicas tiene efectos en los procesos biológicos y geofísicos del océano e impacta los ecosistemas. El cambio global en el medio oceánico se manifiesta, entre otros fenómenos, a través del calentamiento y la acidificación del océano, esta última incentivada por el incremento de $\mathrm{CO}_{2}$ atmosférico. Para una revisión detallada sobre estos dos fenómenos globales véase a Rojas (2013).

\section{El calentamiento del océano}

El conocimiento sobre el calentamiento de los océanos, así como sobre el estado y la dinámica del medio marino, se ha incrementado aceleradamente en los decenios recientes con los resultados de las mediciones y estimaciones en todo el mundo (Lyman, et al., 2010; IPCC, 2013); igualmente, se ha progresado en el modelado de la incidencia del factor antrópico en el calentamiento del océano (Pierce, et al., 2006). Así mismo, se han hecho observaciones del calentamiento de las aguas profundas del océano, con lo que se han evidenciado cambios en la circulación termohalina global (Fukasawa, et al., 2004), en particular en el océano Atlántico norte, donde el calentamiento del océano tiene grandes implicaciones para el sistema climático global (Dmitrenko, et al., 2008). Con base en estas observaciones se ha podido establecer que el calentamiento de las aguas marinas es global y afecta los $700 \mathrm{~m}$ superiores de la columna de agua. Este fenómeno ha ocurrido a un ritmo cercano a $0,1^{\circ} \mathrm{C}$ por decenio durante los últimos 40 años y se prevé que hacia finales del siglo XXI haya alcanzado entre $0,6 \mathrm{y}$ $2,0^{\circ} \mathrm{C}$ en la capa superior de 100 metros (IPCC, 2013).

El calentamiento de las aguas marinas ha tenido diversos efectos en las comunidades ecológicas marinas, en particular en los organismos y ecosistemas marinos, costeros y estuarinos (Kordas, et al., 2011; Madeira, et al., 2012). Los arrecifes coralinos son los ecosistemas marinos más propensos a verse afectados por el aumento de la temperatura del mar, lo cual disminuye su resistencia al blanqueamiento, ocasionando la pérdida de cobertura y la disminución de tasas de crecimiento en algunas zonas (Brierley \& Kingsford, 2009; Instituto de Investigaciones Marinas y Costeras "José Benito Vives de Andréis" -Invemar, 2009; Lough \& Cooper, 2011; Putnam \& Edmunds, 2011). Los corales se desarrollan normalmente entre los 17 y los $34{ }^{\circ} \mathrm{C}$, pero la mayoría vive dentro de su límite máximo; por lo tanto, los cambios en la temperatura pueden deteriorar sus especies y comunidades (Díaz, et al., 2000). Igualmente, las praderas de pastos marinos se desarrollan bien entre los 28 y los $30{ }^{\circ} \mathrm{C}$, y la temperatura límite es de $37^{\circ} \mathrm{C}$, por lo que las alteraciones bruscas y prolongadas de la temperatura pueden afectarlos (Díaz, et al., 2003). Otro de los ecosistemas más afectados por el calentamiento del océano son los manglares, según lo han establecido Brierley \& Kingsford (2009) y Gomes-Soares, et al. (2012), quienes detectaron cambios en la solubilidad química, la disponibilidad de nutrientes y la degradación de la materia orgánica que afectaban la cadena trófica y los organismos marinos asociados a este ecosistema.

\section{La acidificación del océano}

En la actualidad se dispone de conocimiento sobre la distribución espacial global del $\mathrm{pH}$ y se han hecho estimaciones de la acidificación de los océanos del mundo (Feely, et al., 2009). Además de usar los datos de mediciones 
puntuales efectuadas en diversos períodos y lugares, para el conocimiento de la distribución espacial del $\mathrm{pH}$ y el estudio de la acidificación del océano se han reconstruido series de datos de $\mathrm{pH}$ a partir de la temperatura superficial del mar (TSM) y la clorofila- $a$ en el Pacífico norte (Nakano \& Watanabe, 2005).

$\mathrm{El} \mathrm{pH}$ del agua de mar está relacionado con diversos factores, entre los cuales las concentraciones de $\mathrm{CO}_{2}$ atmosférico y de $\mathrm{CO}_{2}$ disuelto en el agua desempeñan un papel importante. La absorción oceánica de $\mathrm{CO}_{2}$ atmosférico proveniente de la quema de combustibles fósiles podría generar grandes cambios del $\mathrm{pH}$ en el agua de mar en los próximos siglos, comparado con los registros geológicos de los pasados 300 millones de años (Caldeira \& Wickett, 2003). Igualmente, Khatiwala, et al. (2009) sugieren que la absorción de $\mathrm{CO}_{2}$ antropogénico por parte del océano desde la revolución industrial ha aumentado notablemente a partir de 1950 y que la biosfera terrestre ha sido una fuente neta de $\mathrm{CO}_{2}$ durante este periodo, lo que también contribuye a su absorción por el océano. Según el IPCC (2013), desde la revolución industrial el $\mathrm{pH}$ ha disminuido en 0,1 unidades, tendencia que en los últimos decenios se ha mantenido con un ritmo de $-0,0014$ a -0,0024 por año. Según Marsh (2005), el pH podría disminuir en 0,5 unidades hacia el 2100 , no obstante, el IPCC (2013) señala que tal reducción podría estar entre 0,065 y 0,31 unidades.

Gledhil, et al. (2008) han encontrado que el proceso de acidificación en el Caribe en el período de 1998 al 2006 generó una fuerte disminución del estado de saturación de la aragonita en la región. Asimismo, con base en un modelado, Friedrich, et al. (2012) señalaron que ya a comienzos del siglo XXI el proceso de acidificación del océano ha provocado que la señal de la reducción del estado de saturación de la aragonita sobrepase en cinco veces su variabilidad natural.

La acidificación del océano ha tenido impacto en los ecosistemas marinos, incluidos los corales tropicales constructores de arrecifes, los corales de agua fría, las algas coralinas incrustantes y los pastos marinos, entre otros, ocasionando cambios en sus funciones (Widdicombe $\&$ Spicer, 2008; Kleypas \& Yates, 2009). La acidificación del océano ha afectado los arrecifes coralinos, causando la reducción de su biodiversidad, el blanqueamiento y, en algunas ocasiones, su muerte (Crabbe, 2009; Veron, et al., 2009), así como la disminución del crecimiento del esqueleto (Krief, et al., 2010), la reducción de la densidad de las zooxantelas (Anlauf, et al., 2011), la disminución de la calcificación (Iguchi et al., 2012) y cambios en las comunidades marinas asociadas (Ishimatsu, et al., 2008). No obstante, Idso \& Ferguson (2009) señalan que, aunque durante el siglo pasado el $\mathrm{pH}$ disminuyó en 0,1 unidades, las tasas de calcificación de los corales ha aumentado, al igual que la temperatura y la concentración de $\mathrm{CO}_{2}$, por lo tanto, la disminución potencial del pH oceánico no parece haber causado un detrimento en los corales y otras formas de vida marina.

La acidificación del océano también ha afectado las comunidades de macroalgas, disminuyendo la riqueza de algunas especies y alterando sus hábitats (Porzio, et al., 2011). Las praderas de pastos marinos también pueden verse afectadas por la acidificación del océano, cuyo impacto varía según la especie y las condiciones ambientales y causa perturbación en la distribución al alterar la competencia entre las especies de pastos marinos y entre sus poblaciones y las de algas (Waycott, et al., 2007; Brierley \& Kingsford, 2009).

La acidificación del océano afecta igualmente a los ecosistemas de manglares, ya que la reducción del $\mathrm{pH}$ incide en la solubilidad química, la disponibilidad de nutrientes, la descomposición de la materia orgánica, la mineralización diagenética de la materia orgánica, y ocasiona alteraciones en la cadena trófica de los estuarios asociados a este ecosistema (Comeaux, et al., 2012; Jennerjahn, 2012).

\section{El ascenso del nivel medio del mar}

Otro fenómeno asociado al cambio global en el océano es el ascenso del nivel medio del mar, el cual ha sido del orden de 1 a $2 \mathrm{~mm}$ por año durante el siglo XX, más exactamente 1,7 \pm 0,2 mm según lo señala el IPCC (IPCC, 2013), debido principalmente a la expansión térmica del agua y a su intercambio con la criosfera; además, la circulación oceánica y la presión atmosférica pueden producir cambios regionales en la magnitud y el signo diferentes al cambio global. El IPCC (2013) proyecta un aumento de 0,25 a 0,82 metros hacia el 2100 en comparación con lo observado entre 1961 y 1990 . Este ascenso relativamente acelerado del nivel medio del mar afectará ecosistemas marinos como los estuarios y los manglares, ya que, entre otros efectos adversos, se produciría la inundación de los suelos causando la muerte de la flora por estrés salino. También los sistemas de playa y los sistemas humanos asentados en las costas se verán afectados por el avance paulatino del nivel del mar (Kennedy, et al., 2002), y dado que los estuarios y manglares contribuyen a la productividad biológica de los sistemas costeros, su agotamiento o su pérdida podría afectar el flujo de nutrientes, la circulación de energía y el hábitat esencial de gran cantidad de especies (Hoegh-Guldberg, 2005).

\section{Los ecosistemas marinos y costeros y sus bienes y servicios}

Los ecosistemas marinos y costeros ofrecen bienes y servicios esenciales a la sociedad y están siendo afectados (positiva o negativamente) por el cambio global, ya que la alteración de los patrones de las corrientes y la disposición de nutrientes modifica la productividad y transforma, en general, organismos y ecosistemas marinos, afectando de diversas formas las poblaciones humanas costeras. A su vez, este impacto en el ambiente marino puede ocasionar problemas económicos y sociales en las regiones costeras, 
pues afecta las pesquerías, la comercialización de recursos marinos, el turismo, la acuicultura y la identidad cultural, entre otros (Cooley, et al., 2009; Madin, et al., 2012). Es importante tener en cuenta que el impacto en los bienes y servicios de los ecosistemas marinos no solo se debe al cambio global; existen otros factores de alteración como la explotación intensiva de los recursos marinos, la contaminación del ambiente acuático, los eventos climáticos extremos, los métodos inapropiados de pesca, la modificación de los sistemas bióticos por parte de los humanos y el turismo excesivo, entre otros (Martínez, et al., 2007; Mooney, et al., 2009; Laurans, et al., 2013). De acuerdo con Malone, $\boldsymbol{e t}$ al. (2010), los bienes y servicios de los ecosistemas marinos se ven especialmente afectados por el calentamiento y la acidificación del océano, que afectan la regulación natural y la calidad del agua, la productividad primaria, el hábitat de la biodiversidad, los ciclos biogeoquímicos y la salud de los habitantes. El aumento de la temperatura y la disminución del pH en el océano generan el desplazamiento de las especies demersales y pelágicas hacia los polos, reduciendo las poblaciones de peces locales, afectando la pesca y, por lo tanto, la economía de las regiones costeras en el mundo (Perry, et al., 2010b). El deterioro de los ecosistemas marinos como los arrecifes coralinos, las praderas de pastos marinos y los manglares causado por el calentamiento y la acidificación del océano tiene un gran impacto en los organismos asociados, reduciendo la población de organismos de uso comercial, lo cual incide en la economía de las regiones costeras, pues su pesca representa el sustento de la mayoría de sus habitantes (Cooley, et al., 2009; Remoundou, et al., 2009; Barange, et al., 2010; Barbier, 2012; Madin, et al., 2012), afectando la seguridad alimentaria y la generación de empleo en las regiones costeras (Cooley, et al., 2009; Perry, et al., 2010b; Madin, et al., 2012).

Dada la magnitud del impacto que los cambios en el medio marino producen en la economía mundial, el tema pasó de ser de interés netamente científico a constituirse en una preocupación para los sectores económicos y políticos de diferentes países, lo que ha impulsado la realización de estudios aplicados en sectores y regiones específicas, con el fin de disponer de estrategias para la mitigación y la adaptación al cambio global futuro (McNeil, 2006). Se han llevado a cabo estudios sobre el manejo de las pesquerías y su adaptación al cambio global (Grafton, 2010; Arnason, 2012; Ruckelshaus, et al., 2013); la sinergia entre la gobernanza y la ciencia integral para facilitar el desarrollo de una política en pesquerías más efectiva (Miller, et al., 2010); el manejo socioecológico de los ecosistemas marinos (Barange, et al., 2010; Perry, et al., 2010a; Perry, et al., 2010b), y sobre la inclusión de la actividad humana en el manejo de los ecosistemas marinos (Barange, et al., 2010), entre otros.

En Colombia el conocimiento sobre el tema aún es incipiente, razón por la cual se exploran aquí los cambios en la temperatura de la superficie del mar y del $\mathrm{pH}$ a largo plazo en el sector de la zona costera del mar Caribe y el océano Pacífico colombianos.

\section{Área de estudio}

El área de estudio se concentró en la franja costera de las áreas marítimas colombianas, las cuales representan el $48 \%$ del territorio colombiano y se localizan en el mar Caribe y el océano Pacífico (Figura 1). La zona de uso económico exclusivo en el mar Caribe tiene $1.800 \mathrm{~km}$ de línea costera y un área superficial aproximada de $589.560 \mathrm{~km}^{2}$, mientras que en el océano Pacífico ésta tiene $1.300 \mathrm{~km}$ de línea costera y un área superficial de $339.100 \mathrm{~km}^{2}$, aproximadamente (Instituto de Hidrología, Meteorología y Estudios Ambientales de Colombia, IDEAM, 1998; Departamento Nacional de Planeación, DNP, 2007).

En las áreas marítimas colombianas se presenta una rica diversidad de ecosistemas marinos y costeros, incluidas lagunas costeras, estuarios, arrecifes de coral, manglares, praderas de pastos marinos, costas arenosas, litorales rocosos $\mathrm{y}$ fondos sedimentarios. Las lagunas costeras y los manglares se consideran ecosistemas costeros y las praderas de pastos marinos y las áreas coralinas, ecosistemas bénticos marinos (IDEAM-IGAC-IAVH-INVEMAR-I.SINCHI-IIAP, 2007). Entre las clasificaciones de los ambientes marinos y costeros colombianos basadas en sistemas de clasificación y regionalización internacionalmente reconocidos, se destaca la establecida por el Instituto de Hidrología, Meteorología y Estudios Ambientales, IDEAM, el Instituto Geográfico Agustín Codazzi, IGAC, el Instituto de Investigación de Recursos Biológicos Alexander von Humboldt, IAvH, el Instituto de Investigaciones Ambientales del Pacífico Jhon von Neumann, IIAP, el Instituto de Investigaciones Marinas y Costeras José Benito Vives De Andréis, Invemar, y el Instituto Amazónico de Investigaciones Científicas, SINCHI (2007).

\section{Materiales y métodos}

Con el fin de establecer la expresión regional e inferir los posibles impactos en el medio marino y costero de los fenómenos marinos globales asociados al calentamiento y acidificación del océano, se definieron las variables para los ámbitos global y regional, y para los impactos. Estos fenómenos se representaron con las variables de temperatura superficial del mar y $\mathrm{pH}$.

Para establecer las posibles condiciones futuras de la temperatura de la superficie del mar (TSM) y el pH, se procedió de la siguiente manera: a) se establecieron las relaciones de estas variables con la temperatura del aire (TSA) y las concentraciones de $\mathrm{CO}_{2}$ atmosférico; b) se hallaron los valores de TSA y de $\mathrm{CO}_{2}$ futuros para el mar Caribe y el océano Pacífico a partir de lo planteado por el IPCC (2000) en los escenarios A2 y B2, y c) utilizando las ecuaciones establecidas inicialmente y los valores futuros de TSA y de $\mathrm{CO}_{2}$, se calcularon la TSM y el $\mathrm{pH}$ futuros para las dos regiones. 

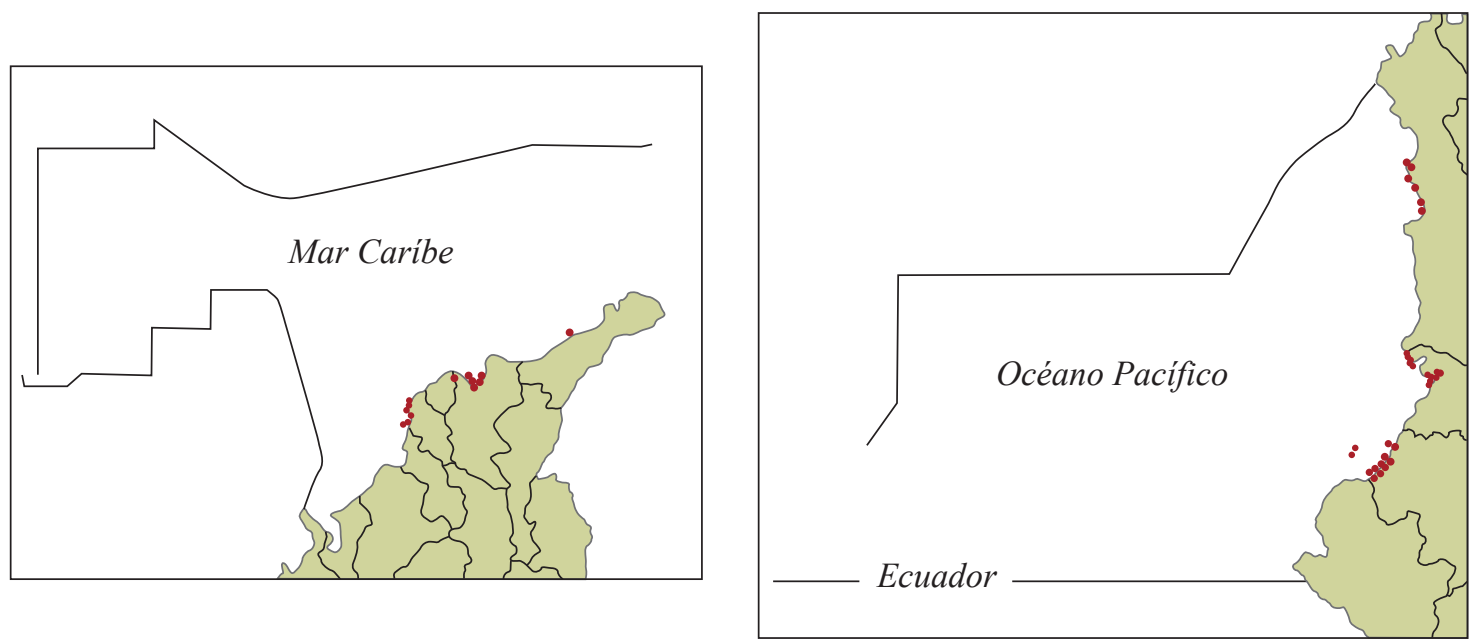

Figura 1. Área de estudio con la localización de los puntos con registros de la temperatura de la superficie del mar y del pH en el mar Caribe (izquierda) y en el océano Pacífico (derecha) colombianos.

Una vez establecida la magnitud de los posibles cambios en la TSM y el pH en el 2025, 2050, 2075 y 2100 para los sectores del Caribe y del Pacífico bajo estudio, se infirieron los impactos que dichas condiciones podrían tener en los ecosistemas marinos costeros de las respectivas regiones.

\section{Datos e información}

La información sobre la TSM y el pH para el Caribe colombiano fue suministrada en archivo digital por el Invemar, cuyos datos fueron recolectados en diferentes estaciones costeras, oceánicas y estuarinas en el período 1993-2011. Para el Pacífico, los datos de las variables mencionadas se tomaron del Sistema de Información de la Red de Vigilancia de la Calidad Ambiental Marina de Colombia (REDCAM) para el periodo 1993-2011 mediante consulta en la página de internet del Invemar. Los sitios sobre los que se dispone de información se distribuyeron según se muestra en los mapas de la Figura 1.

El IDEAM facilitó los datos sobre la temperatura mensual del aire y la TSM en algunos sitios del área de estudio durante el periodo 1993-2011. Los datos de concentración de $\mathrm{CO}_{2}$ atmosférico se tomaron de la base de datos de la National Ocean and Atmosphere Administration (NOAA) para el mismo periodo, disponible sin costo en el sitio ftp:// ftp.cmdl.noaa.gov/ccg/co2/trends/co2_annmean_mlo.txt.

Los valores futuros de la TSA se obtuvieron de los escenarios elaborados por Ruiz (2010) y Pabón (2012), y los datos de las futuras concentraciones de $\mathrm{CO}_{2}$ se obtuvieron del informe de escenarios del IPCC (2000).

\section{Organización y procesamiento de datos}

La información procedente de la base de datos del Invemar se organizó en una tabla que incluyó la fecha (día-mes-año), la latitud y la longitud, la TSM y el pH. Se calcularon los promedios diurnos (entre 12:00 m y 5:00 pm), y los nocturnos (0 y 5 am), para así estimar los promedios diarios. Estos datos se verificaron con el fin de construir los promedios anuales por área (mar Caribe u océano Pacífico).

Los procesos de largo plazo asociados al cambio global se analizaron a partir de los promedios anuales de la TSM por área (Caribe y Pacífico), filtrando así la estacionalidad y la variabilidad espacio-temporal de alta y media frecuencia para enfocarse en los procesos de largo plazo.

Al verificar las series de datos anuales, se encontró que en el período 1993-1995 los valores de la TSM y del pH fueron atípicamente altos y no se mantuvieron dentro del rango característico de las dos variables para la región; por ello en el análisis se consideraron solamente los datos a partir de 1996.

\section{Metodología para el análisis de las tendencias y el establecimiento de las relaciones entre variables}

Para el análisis de tendencias de largo plazo se calcularon las tendencias lineales en las dos variables con base en los datos anuales del período 1996-2011. Con las series de datos anuales de las dos variables se exploraron las relaciones entre TSM y TSA, pH y TSM y pH y $\mathrm{CO}_{2}$, con el fin de estimar los cambios que podrían ocurrir en la TSM y en el pH debido a los cambios en la TSA y en las concentraciones de $\mathrm{CO}_{2}$ atmosférico, los cuales generarían diferentes escenarios climáticos. Para el establecimiento de la relación entre $\mathrm{pH}$ y TSM se exploraron diversas formas de regresión (lineal y polinomial) entre las variables correspondientes.

La relación entre TSM y TSA se calculó utilizando la temperatura media anual del aire por área (calculada a partir de datos suministrados por el IDEAM), y la TSM media anual por área, cálculo que se utilizó en la estimación de la magnitud del calentamiento futuro. 
En cuanto a la relación entre $\mathrm{pH}$ y TSM, se exploró la dependencia del $\mathrm{pH}$ de la TSM utilizando las series de valores anuales medios por área de la TSM y las de los valores anuales de $\mathrm{pH}$ medio por área.

Para establecer la relación entre $\mathrm{pH}_{\text {y }} \mathrm{CO}_{2}$ se tomaron los datos de las concentraciones de $\mathrm{CO}_{2}$ en la atmósfera medidas en la estación de Mauna Loa (Hawái), a los cuales se accedió a través de la página ftp://ftp.cmdl.noaa.gov/ccg/co2/trends/ co2_annmean_mlo.txt.

\section{Sobre la calibración, la validación y el ajuste de los modelos de relación}

Las relaciones polinomiales obtenidas como modelo arrojaron resultados que diferían de la realidad, por lo cual fue necesario tratarlas para acercar los resultados de los modelos de regresión a la realidad reflejada por los datos registrados.

Inicialmente, se hizo una calibración que consistió en sintonizar manualmente la constante de cada ecuación hasta obtener valores cercanos. También se aplicó un coeficiente $k_{\text {ajuste }}($ ecuación 1):

$$
k_{\text {ajuste }}=\frac{p H_{\text {observado }}}{p H_{\text {mod lado }}}
$$

Así, los resultados obtenidos del modelo se ajustaron de la siguiente manera (ecuación 2):

$$
p H_{\text {modelado }}^{\text {ajustado }}=k_{\text {ajuste }} * p H_{\text {modelado }}
$$

La validación se efectuó con base en el cálculo del error cuadrático medio (RMSE) obtenido de la comparación de los valores de $\mathrm{pH}$ modelados con los valores observados.

\section{Método de estimación y análisis de los posibles cambios futuros}

La posible magnitud del calentamiento del mar hacia el 2050 y el 2100 se estimó utilizando los escenarios A2 y B2 de calentamiento de la atmósfera sobre la región obtenidos por Ruiz (2010) y Pabón (2012), y aplicando la relación establecida entre la TSA y la TSM descrita en el punto anterior. Tal relación supone que el calentamiento de la atmósfera induce un aumento de la TSM dados los cambios que habría en los flujos que desde la atmósfera van hacia la superficie, en particular la radiación de onda larga, el calor sensible y el calor latente; los cambios en la cantidad de radiación de onda larga incidente estarían asociados a la modificación de la humedad del aire o de la nubosidad.

La tarea de establecer con exactitud la proporción del calentamiento de la superficie del mar debido al calentamiento de la atmósfera es compleja y está fuera de los alcances del presente trabajo. Por ello, para efectos de este trabajo se utilizó la diferencia directa entre TSM y TSA, que según Jackson \& Wick (2010) se ubica con mayor frecuencia entre los 0,5 y $0,6^{\circ} \mathrm{C}$ (un análisis similar se ha publicado en http://bobtisdale.blogspot.com/2010/12/toa-project-seaair-and-sea-surface.html). No obstante, tal diferencia tiene particularidades regionales (ver, por ejemplo, a Galbraith, et al., 2012), por lo que aquí se analizaron las diferencias a partir de los datos anuales de TSM y de TSA obtenidos para el Caribe y el Pacífico colombianos.

Para estimar los posibles cambios futuros del $\mathrm{pH}$ regional, se utilizó la relación establecida entre pH y TSM en la que se introdujeron los cambios de la TSM que los escenarios A2 y B2 generarían hacia el 2050 y el 2100 (IPCC, 2000) en las concentraciones de los gases de efecto invernadero.

\section{Determinación del impacto en los ecosistemas marinos}

Este trabajo se enfocó en los ecosistemas marinos más sensibles a los cambios de las características físicas y químicas del océano, como son los arrecifes coralinos, las praderas de pastos marinos y los manglares (Kennedy, $\boldsymbol{e t}$ al., 2002; Fischlin, et al., 2007).

El estudio del impacto de los cambios futuros de la TSM sobre los ecosistemas marinos colombianos se efectúo a partir de situaciones análogas pasadas, que están suficientemente documentadas. En el caso del océano Pacífico, por ejemplo, los eventos de El Niño y La Niña son análogos de calentamiento y de enfriamiento, respectivamente. Para el Caribe se analizaron las descripciones publicadas sobre el efecto de las condiciones especiales en el campo térmico en los ecosistemas regionales.

El impacto de los cambios en el $\mathrm{pH}$ en los ecosistemas marinos se infirió a partir de información secundaria sobre los resultados de investigaciones efectuadas hasta ahora por diferentes autores. Igualmente, el impacto de estos fenómenos del cambio global en el ámbito socioeconómico de los ecosistemas marinos seleccionados, se analizó a partir de reportes y publicaciones especializados.

\section{Resultados y discusión}

A continuación se exponen los resultados del análisis de las tendencias de largo plazo en las variables de estudio; se presenta la cuantificación de las relaciones entre las variables, con lo que se buscaba estimar condiciones futuras; se plantean los escenarios de cambio en la TSM y en el pH, y finalmente se señalan cuáles podrían ser los impactos de los cambios en los ecosistemas marinos.

Comportamiento de la temperatura superficial del mar y el pH en el mar Caribe y el océano Pacífico colombianos

El comportamiento de la TSM y el pH expresado en promedios anuales por área en el mar Caribe y el océano Pacífico colombianos se presenta en la Figura 2 a y b, respectivamente (ver síntesis en la Tabla 1).

Las tendencias de largo plazo de la TSM (Figura 2a) mostraron en los dos casos calentamiento cercano a los $0,2{ }^{\circ} \mathrm{C}$ por decenio, siendo un tanto mayor en el mar Caribe $\left(0,23{ }^{\circ} \mathrm{C}\right.$ por decenio) que en el océano Pacífico $\left(0,18{ }^{\circ} \mathrm{C}\right.$ por decenio), lo que coincide con las tendencias mundiales 
a) Caribe Temperatura de la superficie del mar

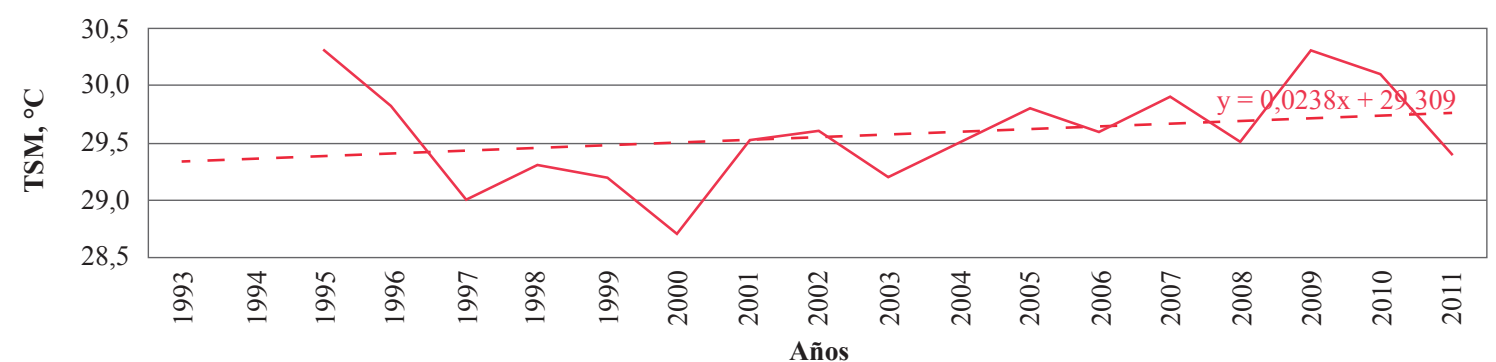

Pacífico Temperatura de la superficie del mar

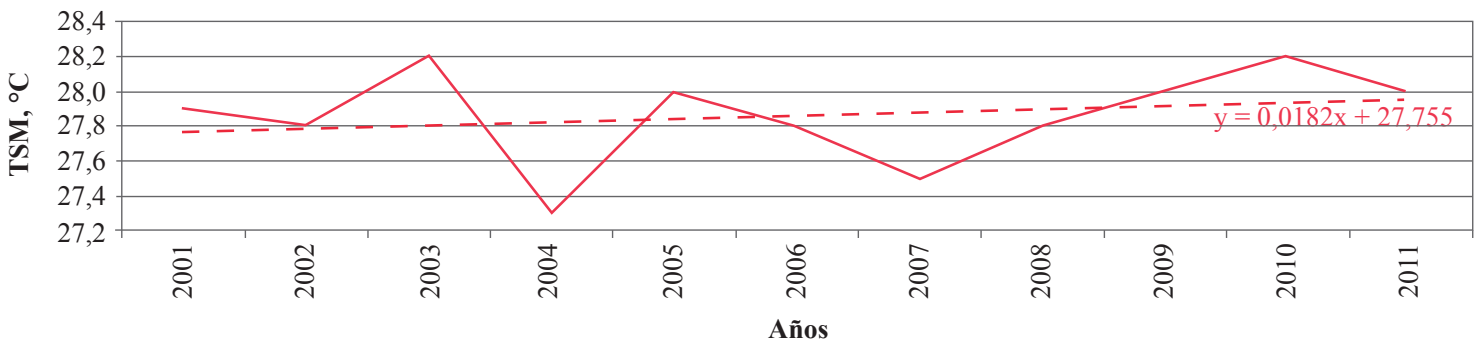

b) Caribe $\mathrm{pH}$ del agua del mar

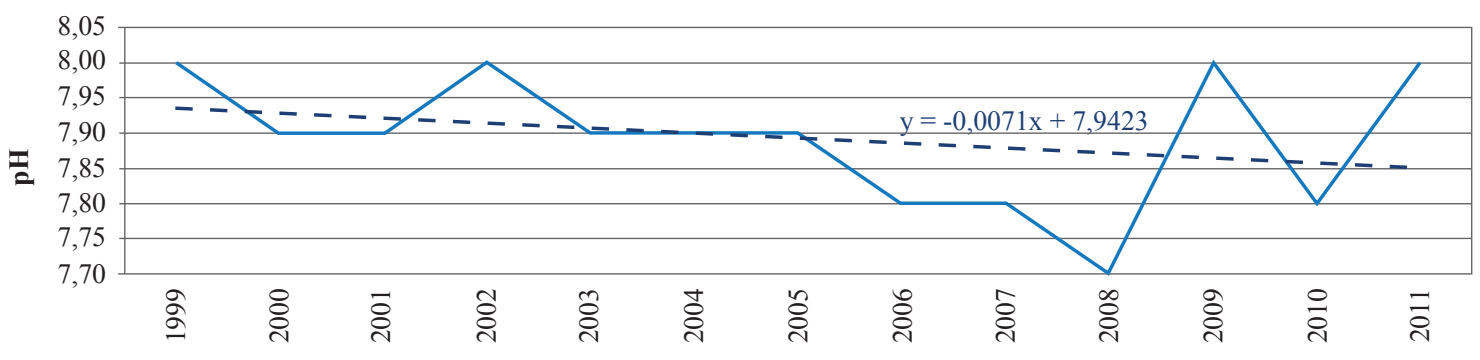

Pacífico $\mathrm{pH}$ del agua del mar

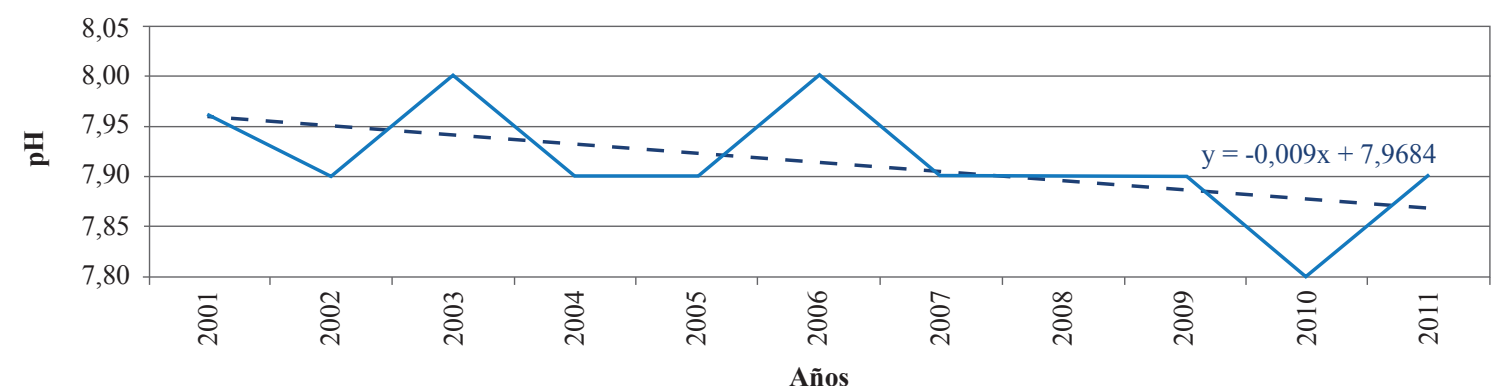

Figura 2. Comportamiento de la temperatura media anual del mar (a) y del pH (b) en el Caribe y el Pacífico colombianos

(Lyman, et al., 2010). La tendencia de largo plazo de los promedios anuales de $\mathrm{pH}$ por área (Figura $2 \mathrm{~b}$ ) evidenció que en el mar Caribe esta variable está disminuyendo en un rango de $-0,0071$ unidades por año, lo que indica un ritmo de acidificación mayor que el promedio mundial (entre -0,015 y -0,024 unidades por decenio, según el IPCC, 2013). En el área marítima de Colombia en el océano Pacífico la tendencia del pH es negativa (-0,009 unidades por año), igual o ligeramente mayor que el promedio global. En los dos casos la tendencia negativa indica una acidificación del océano que es mayor en el océano Pacífico que en el mar Caribe.

\section{Relaciones entre el pH y la temperatura superficial del mar}

La Figura 3a presenta los modelos de la relación entre el pH y la TSM para el mar Caribe y el océano Pacífico colombianos. En los dos casos el modelo que mejor se ajustó $\left(\mathrm{R}^{2}=0,65\right.$ y $\left.\mathrm{R}^{2}=0,91\right)$ fue el basado en el polinomio de quinto orden.

El modelo para el mar Caribe se calibró modificando la constante que aparece al final de la fórmula del polinomio de quinto orden (azul en la Figura 3a), como se expresa en la siguiente ecuación: 
Tabla 1. Tendencia por decenio de la temperatura superficial del mar (TSM) y el pH en el mar Caribe y en el Océano Pacífico colombianos

\begin{tabular}{ccc}
\hline Variable & Tendencia por decenio en el mar Caribe & Tendencia por decenio en el océano Pacífico \\
\hline $\mathrm{TSM}$ & $0,23^{\circ} \mathrm{C}$ & $0,18^{\circ} \mathrm{C}$ \\
$\mathrm{pH}$ & $-0,071$ & $-0,090$ \\
\hline
\end{tabular}
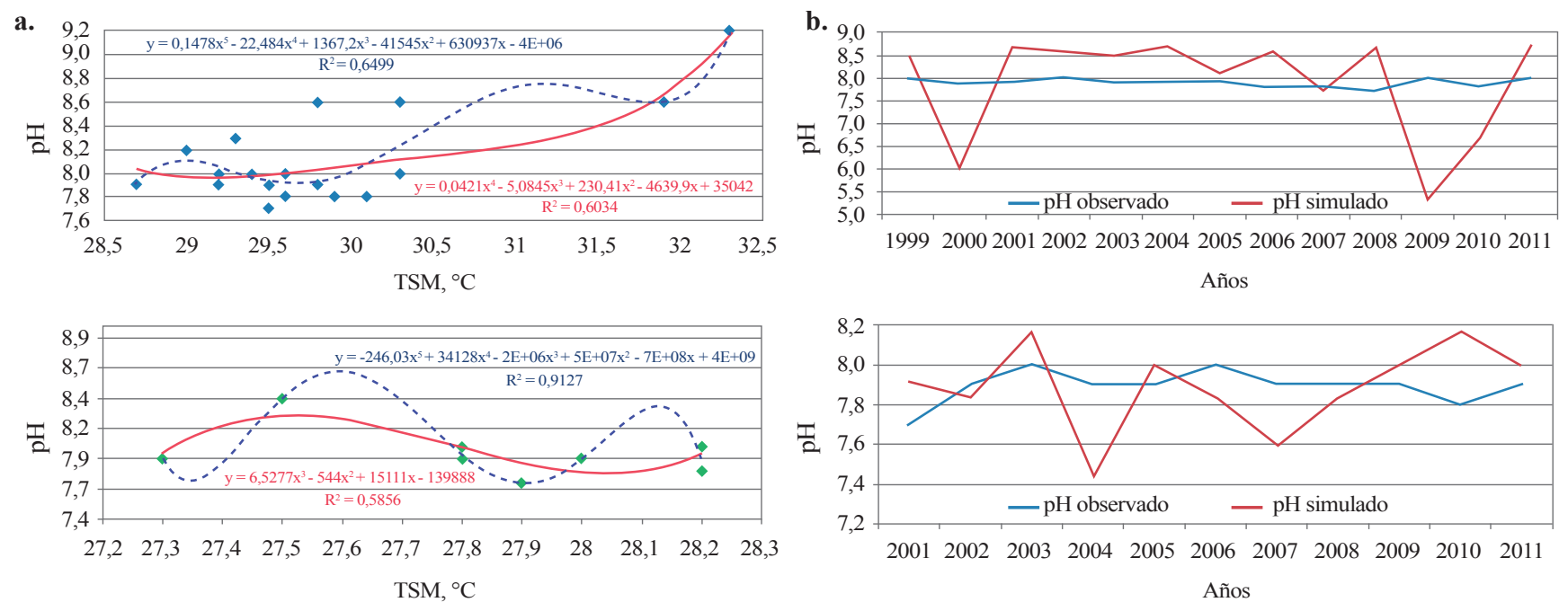

Figura 3. a) Modelos de relación entre la temperatura media anual del mar y el pH medio anual en el mar Caribe (arriba) y el Pacífico (abajo) colombianos (el color de las líneas corresponde al color de los polinomios señalados en la gráfica). b) Comparación de las series de los valores de pH observados y de los modelados para el mar Caribe (arriba) y el océano Pacífico (abajo) colombianos

$$
\begin{aligned}
p H(T S M)= & 0,1478 * T_{S}^{5}-22,484 * T S M+1367,2 \\
& * T S M^{3}-41545 * T_{S}^{2}+630937 \\
& * T S M-3831502,738
\end{aligned}
$$

Para el Pacífico, la formula señalada en el gráfico correspondiente de la Figura 3 a se ajustó mediante la aplicación de un coeficiente $k_{\text {ajuste }}=-2,24638^{*} 10^{-9}$, con lo que el modelo para esta región quedó así:

$$
\begin{aligned}
p H(T S M)= & -2,24638 * 10^{-9}\left[-246,03 * \text { TSM }^{5}\right. \\
& +34128 * T_{S} M^{4}-2000000 * \text { TSM }^{3} \\
& +50000000 * T_{S}^{2}-700000000 \\
& * T S M+4000000000]
\end{aligned}
$$

La comparación de los resultados generados por estos modelos y los datos de las observaciones reales (Figura 3b) permitió establecer que para el océano Pacífico lo aproximación fue mejor que para el mar Caribe. Para validar los resultados de los modelos de relación entre $\mathrm{pH}$ y TSM, se compararon los datos de $\mathrm{pH}$ observados y los modelados mediante el cálculo del error cuadrático medio (RMSE). Como resultado se obtuvieron RMSE del orden de 1,11 y 0,29 para los modelos del mar Caribe y el océano Pacífico, respectivamente, lo que representa un $14 \%$ y un 3,6 \% del valor promedio de las series de $\mathrm{pH}$ correspondientes. Con un mayor muestreo se podrá mejorar el modelo y reducir el error a valores por debajo del $10 \%$ para utilizarlo en diversos cálculos en el futuro.
Los resultados de los valores de $\mathrm{pH}$ obtenidos para el Caribe y el Pacífico colombianos mediante estas fórmulas tuvieron un orden de magnitud similar a los valores reales observados en estas dos regiones del océano mundial. En los sectores analizados del mar Caribe, los valores de $\mathrm{pH}$ promedio por área oscilan entre 8,0 y 8,1 , en tanto que en el océano Pacífico, el pH varía entre 7,95 y 8,0. Las ecuaciones (3) y (4) se podrían utilizar, entonces, para hacer cálculos con valores de TSM de otro período y establecer su promedio de $\mathrm{pH}$, aunque habría que tener cuidado con el uso de dichas ecuaciones dado que en los dos casos se presentó una alta sensibilidad a los cambios de la TSM, debido a que los cambios de décimas de grado producen grandes cambios en el $\mathrm{pH}$.

\section{Relación entre la temperatura de la superficie del mar (TSM) y la temperatura del aire en superficie (TSA)}

La relación entre la TSA y la TSM para el mar Caribe y el océano Pacífico colombianos, se muestra en la Figura 4. En el mar Caribe (Figura 4a, arriba), se observó una relación directa entre la temperatura del aire y de la superficie del mar; la diferencia entre las dos variables fue de 1,5$1,6^{\circ} \mathrm{C}$, que se mantuvo prácticamente uniforme a través de los años. Para el Pacífico, la relación fue un tanto más compleja (por la diferencia en la amplitud de la variabilidad interanual de las dos variables debida a la posición de las corrientes marinas y a la localización de las anomalías de la 
(a)
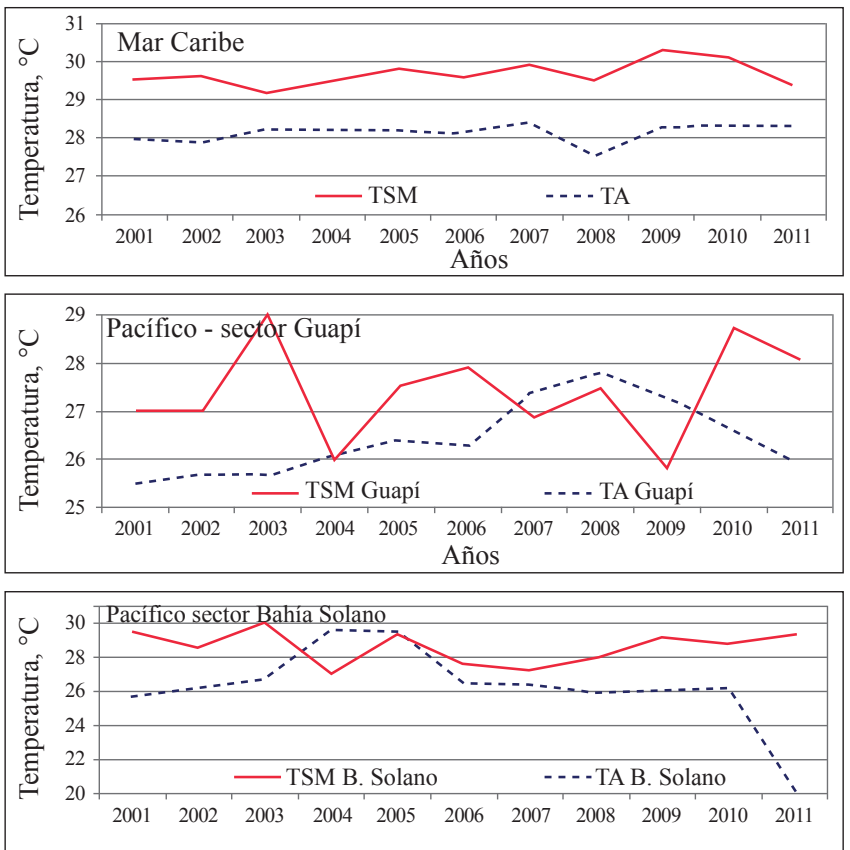

(b)

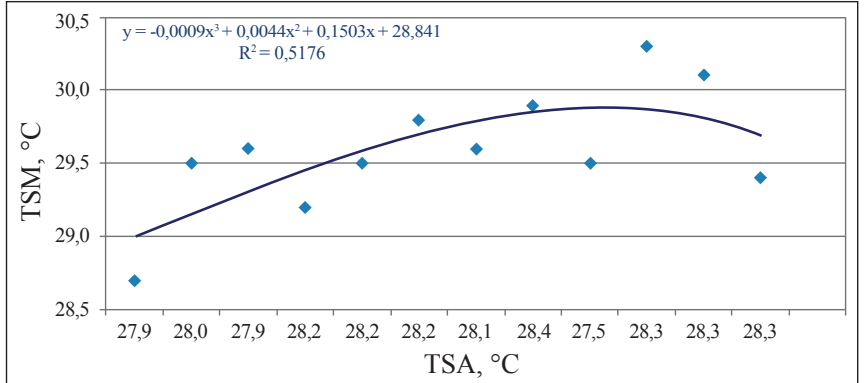

(c)

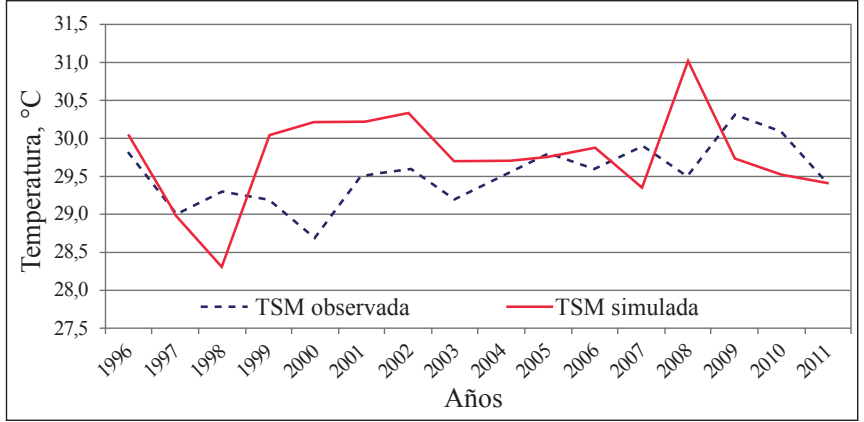

Figura 4. (a) Comparación del comportamiento interanual de la temperatura del aire (TA) y la temperatura superficial del mar (TSM) en el mar Caribe (arriba) y el océano Pacífico (centro y abajo). (b) Cuantificación de la relación existente entre la TSM y la TSA en el Caribe colombiano. (c) Comparación de la TSM observada y la TSM simulada para el área analizada del mar Caribe.

TSM asociadas a los fenómenos de El Niño y de La Niña), y difícilmente se podría establecer un modelo de relación entre la TSM y la TSA para estos sectores del estudio.

La relación entre la TSA y la TSM en el Caribe se cuantificó según se muestra en la Figura 4b, y en ella la TSM se pudo obtener con un alto grado de precisión $\left(\mathrm{R}^{2}=0,72\right)$ empleando la fórmula señalada en el gráfico, la que una vez calibrada y ajustada es la siguiente:

$$
\begin{aligned}
\boldsymbol{T S M}(\boldsymbol{T S} \boldsymbol{A})= & 1,09 *\left[-0,0009 * T S A^{3}-0,0044\right. \\
& \left.* T S A^{2}+0,1503 * T S A+39,5\right]
\end{aligned}
$$

Con esta fórmula se obtuvieron los resultados que se ilustran en la Figura 4c. En este caso se registró un $\mathrm{RMSE}=0,77$, es decir cerca del, 2,5\% del valor promedio de la TSM observada, lo que permitiría elaborar las proyecciones futuras de la TSM a partir de la que se calcule en diferentes escenarios de cambio climático.

Para el océano Pacífico (Figura 4a, centro y abajo), el patrón de la relación entre la TSA y la TSM se vio afectado por la variabilidad interanual asociada a los fenómenos de El Niño y de La Niña (o el ciclo El Niño-Oscilación del Sur, ENOS), siendo ésta más marcada en el sector sur (Guapi), donde la TSM mostró claramente los valores máximos asociados a los fenómenos de El Niño en los periodos 2002-2003 (en el grafico se ve un pico en el 2003) y 2009-2010 (con un pico en el 2010), los cuales no incidieron en el comportamiento de la TSA, pero sí se reflejaron en un aumento de la diferencia entre la TSA y la TSM. También se observaron las condiciones frías que predominaron entre el 2007 y el 2009 asociadas al evento de La Niña; en este periodo la TSA estuvo por encima de la TSM en esta región. En el sector de Bahía Solano el efecto del ciclo ENOS no fue tan marcado y solo se ven los picos asociados a los eventos cálidos (El Niño) cortos de 2002-2003 y de 2004-2005, que no generaron una señal en la TSA. Según se observa en la Figura 4a, abajo, en este sector de Bahía Solano hubo un enfriamiento en el 2004, que tal vez pudo ser un proceso subregional, dado que en ese año no ocurrió el fenómeno de La Niña. En este enfriamiento la TSA se ubicó por encima de la TSM. Por esta diversidad de situaciones en la expresión subregional de la variabilidad interanual, no fue posible establecer un patrón de relación (una ecuación o fórmula) entre la TSM y la TSA para el Pacífico colombiano. Friedrich, et al. (2012) han señalado esta particularidad de la variabilidad interanual del Pacífico oriental (tomando como referencia las Islas Galápagos) en comparación con el Caribe, y plantean que tal heterogeneidad en los procesos influye en las tendencias regionales de la acidificación y en la calcificación en algunos organismos de los ecosistemas marinos.

\section{Relaciones entre el pH del agua marina regional y el $\mathrm{CO}_{2}$ atmosférico global}

La exploración de la relación entre el $\mathrm{pH}$ y el $\mathrm{CO}_{2}$ se hizo con las series de $\mathrm{pH}$ correspondientes a los sectores del mar Caribe y del océano Pacífico analizados y las concentraciones de $\mathrm{CO}_{2}$ medidas en Mauna Loa (Hawaii). En la Figura 5a se 
(a)
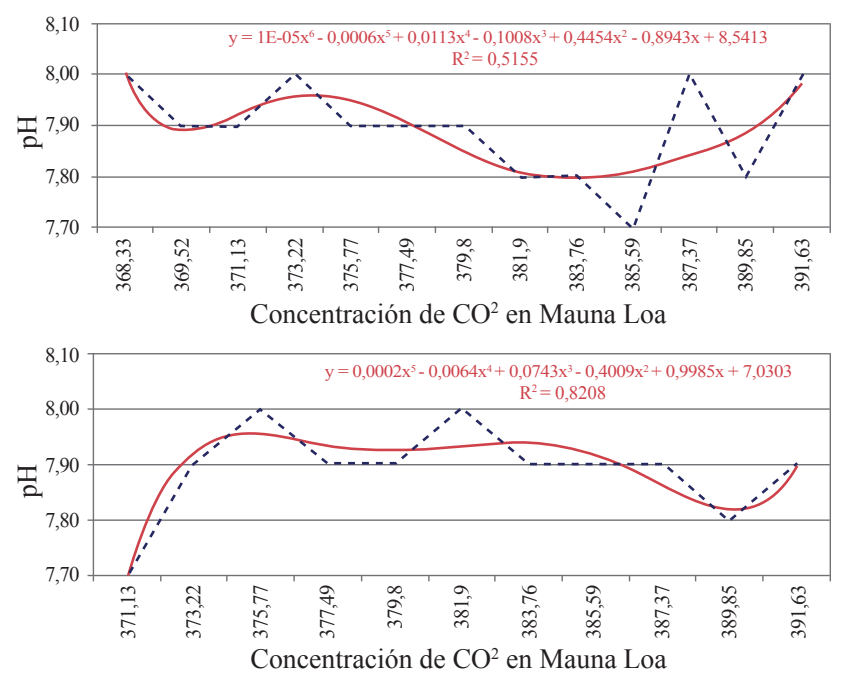

(b)
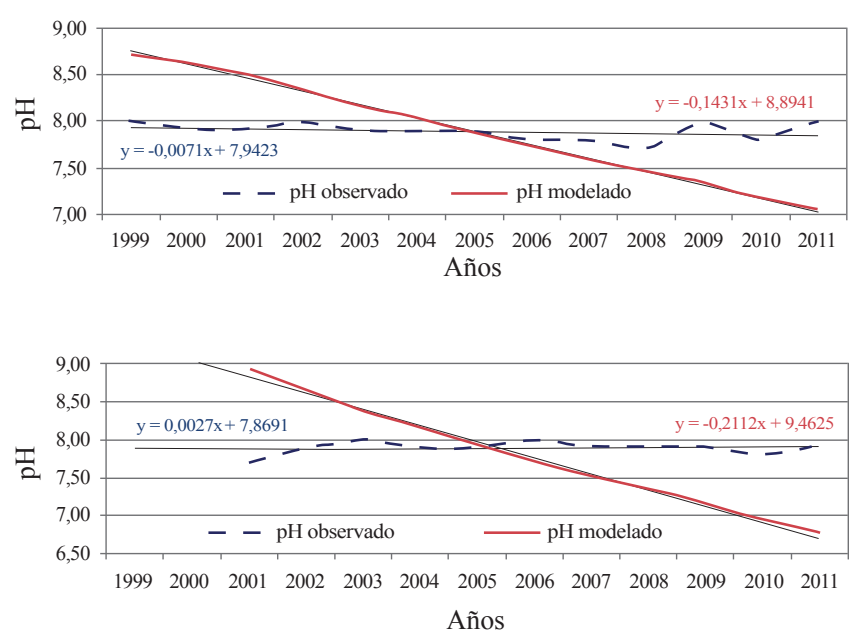

Figura 5. (a) Cuantificación de la relación entre el $\mathrm{pH}$ y el $\mathrm{CO}_{2}$ para el Caribe (arriba) y el Pacífico (abajo) colombianos. (b) Comparación del comportamiento del pH observado y el obtenido a partir de las relaciones (6) y (7) para el Caribe (arriba) y para el Pacífico (abajo).

presenta esta relación en las ecuaciones sobre los gráficos (ecuaciones 6 y 7). Allí se señalan los polinomios con los que se obtuvo el máximo $\mathrm{R}^{2}$ en cada caso.

Una vez calibrados, estos modelos de relación entre el $\mathrm{pH}$ y el $\mathrm{CO}_{2}$ resultaron así:

$$
\begin{aligned}
& \boldsymbol{p H}\left(\boldsymbol{C O}_{2}\right)=3,58184 * 10^{11} /\left[0,00001 * \mathrm{CO}_{2}{ }^{6}-0,0006\right. \\
& * \mathrm{CO}_{2}{ }^{5}+0,0113 * \mathrm{CO}_{2}{ }^{4}-0,1008 * \mathrm{CO}_{2}{ }^{3}+0,4454 * \mathrm{CO}_{2}{ }^{2} \\
& \left.-0,8943 * \mathrm{CO}_{2}+20000000000,541\right] \\
& \text { pH }\left(\boldsymbol{C O}_{2}\right)=11503689098 /\left[0,0002 * \mathrm{CO}_{2}{ }^{5}-0,0064\right. \\
& * \mathrm{CO}_{2}{ }^{4}+0,0743 * \mathrm{CO}_{2}{ }^{3}-0,4009 * \mathrm{CO}_{2}{ }^{2}+0,9985 \\
& \left.* \mathrm{CO}_{2}+7,0303\right]
\end{aligned}
$$

La fuerte tendencia lineal de las concentraciones de $\mathrm{CO}_{2}$ imprimió una tendencia exagerada a la disminución del $\mathrm{pH}$ modelado (Figura 5b). Esto implicó hacer un ajuste de tendencia, que consistió en multiplicar la tendencia de la serie modelada por $3,6 \times 10^{11}$ para el mar Caribe (ecuación 6) y por 1150368908 para el océano Pacífico (ecuación 7). Los promedios de las series de mediciones del $\mathrm{pH}$ para el período analizado en el mar Caribe y en el océano Pacífico fueron de 7,9 en los dos casos; de acuerdo con Doney (2006), el pH es más bajo en el Pacífico que en el Caribe. Los promedios de los modelados fueron de 7,9 y 7,8, respectivamente.

Los resultados obtenidos después del ajuste resultaron similares a los propuestos por otros autores (The Royal Society, 2005; Feely, et al., 2009), lo que sugiere que un incremento de aproximadamente $100 \mathrm{ppmv}$ de $\mathrm{CO}_{2}$ atmosférico conlleva a una reducción de 0,1 unidades de $\mathrm{pH}$ en el agua marina superficial. En el período de 1999 a 2011 analizado, y de acuerdo con las mediciones en Mauna Loa (Hawaii), el incremento del $\mathrm{CO}_{2}$ atmosférico fue del orden de 20 ppmv, lo que generó una disminución del pH de 0,07 unidades por decenio y de 0,09 unidades por decenio en los sectores marino-costeros analizados en el mar Caribe y el océano Pacífico, respectivamente. La aplicación de los modelos ajustados de la relación entre el pH y el $\mathrm{CO}_{2}$ generó tendencias similares.

En las estaciones con ambientes estuarinos también se presenta una acidificación de sus aguas; no obstante, de acuerdo con Feely, et al., (2010) esta acidificación es ocasionada no solo por el proceso global, sino que también puede ser inducida por procesos costeros como la surgencia, rica en $\mathrm{CO}_{2}$, y por los flujos de agua dulce, sedimentos, materia orgánica, nutrientes y contaminantes provenientes del continente. Las tendencias del $\mathrm{pH}$ establecidas son el promedio para la franja marina costera, por lo que incluirían tanto el efecto del proceso global de acidificación como el generado por los procesos regionales. Así las cosas, estas tendencias solo son evidencia de un proceso de acidificación en estas zonas que no puede atribuirse exclusivamente al proceso global. A esto hay que agregar lo señalado por Friedrich, et al., (2012) con relación a la heterogeneidad de los procesos en diferentes regiones.

\section{Los posibles cambios futuros en la temperatura superficial del mar (TSM) y el pH}

En la metodología se propuso que para establecer los cambios futuros de la TSM se usarían los valores de temperatura media anual del aire provenientes de escenarios futuros y su inclusión en el modelo de relación entre la TSM y la TSA establecido. Para los cambios de $\mathrm{pH}$ se utilizaría la relación entre el $\mathrm{pH}$ y la TSA y entre el $\mathrm{pH}$ y el $\mathrm{CO}_{2}$; infortunadamente, la relación entre el $\mathrm{pH}$ y la TSM no 
funcionó lo suficientemente bien, por lo que se optó por estimar el $\mathrm{pH}$ futuro con base en los datos de $\mathrm{CO}_{2}$ de Mauna Loa (Hawaii).

En la tabla 2a se presentan las concentraciones de $\mathrm{CO}_{2}$ bajo los escenarios A2 y B2 del IPCC (2000), mientras que en la tabla $2 \mathrm{~b}$ se presentan los cambios que habría en la TSA en dos

Tabla 2. Valores de las concentraciones de $\mathrm{CO}_{2}$ y de la temperatura media anual del aire proyectados a diferentes períodos del siglo XXI y empleados para el cálculo de escenarios de temperatura media anual de la superficie del mar y del $\mathrm{pH}$ para el Caribe y el Pacífico colombianos. (a) Concentraciones de $\mathrm{CO}_{2}$ atmosférico (en ppmv) para diferentes cortes en el siglo XXI en los escenarios A2 y B2 del IPCC (2000). (b) Cambios en el promedio multianual (norma de 30 años) en la temperatura media anual del aire sobre el Caribe y el Pacífico bajo varios escenarios futuros comparados con la norma observada a finales de siglo XX (1971-2000), según lo establecido por Pabón (2012).

(a)

\begin{tabular}{lcc}
\hline Año & Escenario A2 & Escenario B2 \\
\hline 2025 & 450 & 425 \\
2050 & 550 & 500 \\
2075 & 625 & 550 \\
2100 & 850 & 600 \\
\hline
\end{tabular}

(b)

\begin{tabular}{lcccc}
\hline \multirow{2}{*}{ Región } & \multicolumn{2}{c}{$\mathbf{2 0 1 1 - 2 0 4 0}$} & \multicolumn{2}{c}{$\mathbf{2 0 7 0 - 2 1 0 0}$} \\
\cline { 2 - 5 } & A2 & B2 & A2 & B2 \\
\hline Caribe & 1,5 & 1 & 3 & 2 \\
Pacífico & 1,2 & 1 & 2,5 & 2 \\
\hline
\end{tabular}

ventanas temporales del siglo XXI bajo los escenarios A2 y B2 del IPCC (2000); los datos regionales de la TSA en estos escenarios se extractaron de Pabón (2012).

Con la información de las Tablas 2 a y 2 b y las fórmulas de las relaciones arriba establecidas, se calcularon los cambios que habría en la TSM en los períodos 2011-2040 y 20702100, y los posibles valores futuros del $\mathrm{pH}$ en el 2025, 2050, 2075 y 2100 bajo los escenarios A2 y B2. La referencia de los cambios de la TSM es la norma observada en el periodo de 1971-2000. Los resultados para la TSM en los sectores analizados del mar Caribe y del océano Pacífico se muestran en la Tabla 3a. Es posible constatar que en ambos casos que hacia finales del siglo XXI los valores promedio de la TSM sobrepasarían los $30^{\circ} \mathrm{C}$.

Los resultados para el $\mathrm{pH}$ en diferentes períodos del siglo XXI se exponen en la Tabla 3b. Como se puede apreciar, en el escenario A2 hacia finales del siglo XXI se tendrían valores 0,5 por debajo de los registrados a comienzos del siglo, en tanto que bajo el escenario B2 la reducción del pH sería del orden de 0,3 .

\section{Posible impacto en los ecosistemas marinos colombianos}

Los resultados expuestos sugieren que en el mar Caribe y en el océano Pacífico colombianos hay calentamiento y acidificación de las aguas marinas, lo que constituye una amenaza para sus ecosistemas, en particular los arrecifes coralinos, las praderas de pastos marinos y los manglares. Estos ecosistemas son particularmente sensibles a los cambios en las características físicas y químicas del océano (Kennedy, et al., 2002; Fischlin, et al., 2007).

Tabla 3. Proyecciones de la temperatura media del mar y el pH en el Caribe y el Pacífico para diferentes períodos del siglo XXI. (a) Temperatura media del mar (TSM) bajo los escenarios A2 y B2. (b) pH (en unidades) en el 2025, el 2050, el 2075 y el 2100 bajo los escenarios A2 y B2

(a)

\begin{tabular}{lcccccc}
\hline Región & $\begin{array}{c}\text { TSM promedio en } \\
\mathbf{2 0 0 0 - 2 0 1 0}\end{array}$ & $\mathbf{2 0 1 1 - 2 0 4 0}$ & \multicolumn{2}{c}{$\mathbf{2 0 7 0 - 2 1 0 0}$} \\
\cline { 3 - 6 } & 29,6 & 31,0 & B2 & 30,5 & A2 & 32,0 \\
\hline Caribe & 27,9 & 29,2 & 28,6 & 30,4 & 31,4 \\
Pacífico & & & B2 & 29,8 \\
\hline
\end{tabular}

(b)

\begin{tabular}{|c|c|c|c|c|c|}
\hline \multirow[t]{3}{*}{ Región } & \multirow{2}{*}{$\begin{array}{c}\text { pH promedio en } \\
2000-2010\end{array}$} & \multicolumn{4}{|c|}{ Años } \\
\hline & & 2025 & 2050 & 2075 & 2100 \\
\hline & & \multicolumn{4}{|c|}{ Escenario A2 } \\
\hline Caribe & 7,892 & 7,812 & 7,712 & 7,637 & 7,412 \\
\hline \multirow[t]{2}{*}{ Pacífico } & 7,891 & 7,811 & 7,711 & 7,636 & 7,411 \\
\hline & & \multicolumn{4}{|c|}{ Escenario B2 } \\
\hline Caribe & 7,892 & 7,837 & 7,762 & 7,712 & 7,662 \\
\hline Pacífico & 7,891 & 7,836 & 7,761 & 7,711 & 7,661 \\
\hline
\end{tabular}




\section{Impacto del aumento de la temperatura del mar (TSM)}

Según se estableció en el análisis, el aumento actual de la TSM en el mar Caribe y el océano Pacífico colombianos es de cerca de $0,2{ }^{\circ} \mathrm{C}$, y se proyecta que en el siglo XXI este calentamiento puede alcanzar los $2{ }^{\circ} \mathrm{C}$. Estos cambios indudablemente producirán impactos en los ecosistemas de las áreas marítimas colombianas, los cuales se pueden inferir a partir de la evidencia de los impactos encontrados por diversos autores en otras regiones o para la misma región pero en otros períodos (Alvarado, et al., 1986; Solano, 1994; Díaz, et al., 2000; Díaz, et al., 2003; Vargas-Ángel, et al., 2001; Garzón-Ferreira, et al., 2002; Sheppard \& Rioja-Nieto, 2005; Echavarría-Heras, et al., 2006; Barreto, 2008; Invemar, 2009; Invemar, 2011; Gomes-Soares, et al., 2012). Estos casos análogos pueden ayudar a prever lo que podría ocurrir en la región de análisis. Por ejemplo, en el Pacífico colombiano se ha observado un calentamiento de tal magnitud durante los eventos de El Niño, por lo que, para esta región, lo que ocurre temporalmente en el ambiente marino colombiano en esas condiciones anómalas podría tomarse como escenario para inferir los posibles impactos del calentamiento en el océano en el siglo XXI. La Tabla 4 resume los posibles efectos del calentamiento del mar inferidos a partir de diversas investigaciones llevadas a cabo por diversos autores en el Caribe, el Atlántico tropical occidental y el Pacífico tropical oriental.

Según esta revisión, a medida que transcurra el siglo XXI la cobertura de los arrecifes coralinos colombianos presentaría una paulatina disminución, ya que el aumento de la temperatura del agua reduce su resistencia y su resiliencia, lo que posibilita la reducción de las tasas de calcificación y el blanqueamiento. Esta reducción de los arrecifes coralinos implica una disminución de los organismos y comunidades asociados (Alvarado, et al., 1986; Solano, 1994; Díaz, et al., 2000; Vargas-Ángel, et al., 2001; Garzón-Ferreira, et al., 2002; Sheppard \& Rioja-Nieto, 2005; Invemar, 2009; Invemar, 2011).
El calentamiento del medio marino también propiciará la disminución en la extensión de las praderas de pastos marinos en el mar Caribe colombiano debido a los cambios en sus tasas de crecimiento y funciones fisiológicas y a la reducción de su capacidad fotosintética (Díaz, et al., 2003; Echavarría-Heras, et al., 2006).

Otro de los ecosistemas marinos colombianos impactados por el calentamiento del agua de mar son los manglares, ya que este fenómeno podría ocasionar un aumento en la salinidad y una reducción de los nutrientes, disminuyendo así la productividad primaria del estuario y la densidad de algunas especies de manglar. Estos cambios en el ecosistema significarían, a su vez, una reducción de la abundancia de los organismos asociados (Barreto, 2008; Gomes-Soares, et al., 2012).

\section{Impacto de la disminución del pH}

De acuerdo con los resultados obtenidos, se está presentando una disminución del $\mathrm{pH}$ en el territorio marítimo colombiano del orden de $-0,071$ en el mar Caribe y de $-0,090$ en el océano Pacífico, en tanto que la acidificación del océano podría alcanzar 0,5 unidades a finales del siglo XXI. Estas alteraciones pueden afectar significativamente los ecosistemas marinos de Colombia y podrían deducirse de los estudios de varios autores en diferentes regiones del planeta. La Tabla 5 sintetiza los posibles efectos de la acidificación del océano inferidos a partir de diversas investigaciones realizadas sobre el tema en la región. Sería posible inferir otros efectos a partir de lo que está ocurriendo en otras regiones, pero en la Tabla 5 se incluyen solamente las evidencias en torno a esta región y los resultados de algunos experimentos realizados por investigadores en el tema.

Los ecosistemas de arrecifes coralinos localizados en los mares colombianos podrían ver reducida su biodiversidad durante el siglo XXI, ya que la disminución del pH disminuye

Tabla 4. Posibles efectos del aumento de la temperatura superficial del mar (TSM) en los ecosistemas marinos colombianos

\begin{tabular}{|c|c|c|}
\hline Ecosistema marino costero & Posible efecto & Evidencia a partir de la que se infiere el posible efecto \\
\hline \multirow{3}{*}{ Arrecifes coralinos } & Blanqueamiento de coral & $\begin{array}{l}\text { Blanqueamiento del coral causado por el fenómeno de El Niño (Alvarado, } \\
\text { et al., 1986; Solano, 1994; Díaz, et al., 2000; Vargas-Ángel, et al., 2001; } \\
\text { Garzón-Ferreira, et al., 2002; Invemar, 2009; Invemar, 2011). }\end{array}$ \\
\hline & Pérdida de la cobertura & $\begin{array}{l}\text { Disminución de la cobertura coralina en Colombia (Díaz, et al., 2000; Invemar, } \\
\text { 2009; Invemar, 2011). }\end{array}$ \\
\hline & $\begin{array}{l}\text { Mortalidad de algunas especies } \\
\text { y áreas coralinas }\end{array}$ & Mortalidad de áreas coralinas (Sheppard \& Rioja-Nieto, 2005) \\
\hline \multirow{2}{*}{ Praderas de pastos marinos } & $\begin{array}{l}\text { Disminución de la tasa de crecimiento } \\
\text { y de la cobertura }\end{array}$ & $\begin{array}{l}\text { Disminución de la cobertura ocasionada por el evento de El Niño (Echavarría- } \\
\text { Heras, et al., 2006). }\end{array}$ \\
\hline & $\begin{array}{l}\text { Cambio en las funciones fisiológicas } \\
\text { de las plantas }\end{array}$ & $\begin{array}{l}\text { Cambio en la floración y maduración causado por el evento de El Niño en } \\
\text { Colombia (Díaz, et al., 2003). }\end{array}$ \\
\hline \multirow{2}{*}{ Manglar } & Cambio en la fisiología de las especies & Efecto del calentamiento del océano en los manglares (Gomes-Soares, et al., 2012). \\
\hline & Disminución de la densidad & Manglares en Venezuela (Barreto, 2008) \\
\hline
\end{tabular}


la densidad de las zooxantelas y las tasas de calcificación, ocasionando el blanqueamiento e incluso la muerte de algunas especies. La reducción de la cobertura de los arrecifes de coral significa, a su vez, la disminución de los organismos y comunidades asociados (Anthony, et al., 2008; Bak, et al., 2009; Kleypas \& Yates, 2009; Veron, et al., 2009; Krief, et al., 2010; Anlauf, et al., 2011). Igualmente, la acidificación del océano afectará la distribución de las especies de las praderas de pastos marinos en el mar Caribe colombiano debido a la disminución de su productividad y la competencia con otras especies de pastos marinos y con poblaciones de algas asociadas a este ecosistema (Short \& Neckles, 1999).

Los manglares del territorio marítimo colombiano también serán impactados por la disminución del $\mathrm{pH}$, la cual ocasiona cambios en la solubilidad química, la disponibilidad de nutrientes y la degradación de la materia orgánica, afectando la cadena trófica y los organismos marinos asociados a los estuarios del manglar (Feely, et al., 2010).

\section{Impacto socioeconómico del cambio global en los ecosistemas marinos}

Los posibles efectos socioeconómicos del calentamiento y la acidificación del océano se resumen en la Tabla 6.

Con base en el conocimiento de los impactos registrados y analizados por diferentes investigadores de la región, en especial de instituciones colombianas, es posible inferir los impactos del calentamiento y la acidificación del océano en las regiones costeras del mar Caribe y el océano Pacífico colombianos, los cuales afectarían las pesquerías, la comercialización de recursos marinos, el turismo y otras actividades comerciales.

Tabla 5. Posibles efectos de la disminución del pH en los ecosistemas marinos colombianos

\begin{tabular}{|c|c|c|}
\hline Ecosistema marino costero & Posible efecto & Evidencia a partir de la que se infiere el posible efecto \\
\hline \multirow{5}{*}{ Arrecifes coralinos } & Blanqueamiento & Anthony, et al., 2008 (en general, experimentalmente) \\
\hline & Reducción del crecimiento & Bak, et al., 2009 (corales en Curazao) \\
\hline & $\begin{array}{l}\text { Disminución en la densidad de las } \\
\text { zooxantelas }\end{array}$ & $\begin{array}{l}\text { Disminución de la densidad de zooxantelas en Porites sp y Stylophora } \\
\text { pistillata (Krief, et al., 2010, experimentalmente en condiciones de } \\
\text { acuario controlado) y en Porites panamensis (Anlauf, et al., 2011, } \\
\text { experimentalmente para el Pacífico tropical oriental) }\end{array}$ \\
\hline & Reducción de las tasas de calcificación & $\begin{array}{l}\text { Disminución de las tasas de calcificación en especies de coral } \\
\text { (Anthony, et al., 2008; experimentalmente); Kleypas \& Yates, 2009, } \\
\text { síntesis en la que se citan los corales del Caribe; Krief, } \text { et al., 2010) }\end{array}$ \\
\hline & Mortalidad de algunas especies & $\begin{array}{l}\text { Mortalidad de corales causada por la acidificación del océano } \\
\text { (Veron, et al., 2009). }\end{array}$ \\
\hline \multirow{2}{*}{ Praderas de pastos marinos } & Cambio en la distribución de las especies & $\begin{array}{l}\text { Impacto de la acidificación del océano en pastos marinos (Short \& } \\
\text { Neckles, 1999). }\end{array}$ \\
\hline & Aumento de la productividad & $\begin{array}{l}\text { Aumento de la productividad de Thalassia testudinum en el mar Caribe } \\
\text { (Short \& Neckles, 1999). }\end{array}$ \\
\hline Manglar & $\begin{array}{l}\text { Alteraciones en la cadena trófica y } \\
\text { organismos asociados de aguas estuarinas }\end{array}$ & $\begin{array}{l}\text { Impacto de la acidificación del océano en estuarios de manglares de } \\
\text { Estados Unidos (Feely, et al., 2010). }\end{array}$ \\
\hline
\end{tabular}

Tabla 6. Posibles impactos socioeconómicos identificados en los ecosistemas marinos colombianos

\begin{tabular}{|c|c|c|}
\hline Sector & Posible impacto & Evidencia a partir de la que se infiere el posible impacto \\
\hline \multirow{3}{*}{ Pesca } & $\begin{array}{l}\text { Reducción de la pesca por desplazamiento } \\
\text { de especies comerciales }\end{array}$ & $\begin{array}{l}\text { Migración de plumuda del Pacífico colombiano hacia el norte durante el evento El } \\
\text { Niño } 1997 \text { - } 1998 \text { (Zapata, 2002) }\end{array}$ \\
\hline & $\begin{array}{l}\text { Reducción de las poblaciones de organismos } \\
\text { comerciales }\end{array}$ & $\begin{array}{l}\text { Reducción de especies comerciales en Colombia (Díaz, et al., 2000; Zapata, } \\
\text { 2002; Invemar, 2011) }\end{array}$ \\
\hline & Afectación de la seguridad alimentaria & Impacto socioeconómico de la acidificación del océano (Rodrígues, et al., 2013). \\
\hline \multirow{3}{*}{ Turismo } & Problemas de salud en los turistas & Impacto socioeconómico de la acidificación del océano (Rodrígues, et al., 2013) \\
\hline & Disminución del buceo ecológico y recreacional & Impacto socioeconómico de la acidificación del océano (Rodrígues, et al., 2013). \\
\hline & Efectos económicos en actividades asociadas & $\begin{array}{l}\text { Impacto socioeconómico de la acidificación del océano en ecosistemas marinos } \\
\text { (Rodrígues, et al., 2013). }\end{array}$ \\
\hline
\end{tabular}


En este contexto, es posible prever que el deterioro de los bienes y servicios de los ecosistemas marinos colombianos traería efectos negativos, principalmente en la pesca (artesanal e industrial) y en el turismo, renglones económicos que generan seguridad alimentaria, empleo y sustento económico para los habitantes de estas regiones del país. De acuerdo con la Corporación Colombiana Internacional CCI (2006), en el mar Caribe colombiano se desarrolla la pesca industrial de camarón, atún y pesca blanca, así como la pesca artesanal por parte de comunidades de pescadores en las diferentes regiones; así mismo, en el océano Pacífico se desarrolla la pesca industrial (camaronera, atunera, pesca blanca y pequeños pelágicos), la pesca artesanal y la pesca tradicional de piangua (CCI, 2006; Díaz, et al., 2011).

Dado que en años anteriores se evidenció en el océano Pacífico colombiano la migración de la plumuda, Opisthonema $s p$, hacia el norte del Pacífico durante el evento de El Niño de 1997 - 1998 (Zapata, 2002), lo que disminuyó la abundancia de esta especie, con el calentamiento del agua marina en la región podría darse el desplazamiento definitivo de su hábitat, impactando negativamente y de manera significativa la pesquería artesanal de los habitantes de esta región.

Igualmente, el deterioro de los ecosistemas marinos colombianos debido al calentamiento y la acidificación del océano ocasiona un impacto significativo en los organismos asociados, disminuyendo la población de especies comerciales, lo cual se evidencia con la reducción de la cobertura de los arrecifes de coral (Díaz, et al., 2000), así como de pargos, meros, chernas, langostas, cangrejo y caracoles, entre otros (Díaz, et al., 2000; Invemar, 2011).

El turismo en las regiones costeras del territorio colombiano también podría verse afectado por el calentamiento y la acidificación del océano, ya que ocasionarían problemas de salud en los turistas, disminuirían el buceo ecológico y recreacional y afectarían negativamente la economía de otras actividades asociadas.

\section{Conclusiones}

Según el análisis de las series de datos de la TSM y del $\mathrm{pH}$ recolectadas por el Invemar en diferentes sectores de la zona costera del Caribe y del Pacífico colombiano, se estableció lo siguiente.

- Hay una tendencia al incremento a largo plazo de la temperatura media del mar del orden de $0,23{ }^{\circ} \mathrm{C}$ por decenio en Caribe y de $0,18^{\circ} \mathrm{C}$ por decenio en el Pacífico, lo que corrobora la presencia del calentamiento del océano en estos sectores.

- Hay una tendencia a la disminución largo plazo del pH en los sectores marinos costeros del Caribe y del Pacífico analizados, la cual se estimó en - 0,071 y -0,090 unidades por decenio, respectivamente, lo que evidencia un ritmo de acidificación ligeramente mayor que el promedio mundial. No obstante, dado que algunas estaciones de medición estaban localizadas en ambientes estuarinos, dicha acidificación no se podría atribuir completamente al proceso global, ya que puede ser inducida por procesos que se estén desarrollando en la costa y en el continente.

En lo que se refiere a las condiciones marinas en los sectores de estudio durante el siglo XXI, se encontró que:

- La TSM hacia finales del siglo tendría un aumento de hasta $2{ }^{\circ} \mathrm{C}$ en relación con la observada a comienzos de siglo en los sectores analizados del Caribe y del Pacífico.

- Hacia finales del siglo XXI, en el escenario más drástico de los analizados en este trabajo (A2), el pH habría disminuido hasta en 0,5 sus valores.

Dado que según la síntesis bibliográfica llevada a cabo para el presente trabajo, estos cambios en el ambiente marino son mayores a los que han impactado hasta ahora los ecosistemas en diferentes regiones del mundo, es muy probable que durante el siglo XXI, con la agudización del calentamiento y la acidificación del océano (lo que de acuerdo a Friedrich, et al. (2012), se reducirá la calcificación de los organismos marinos en magnitudes que regionalmente podrían superar hasta en 30 veces la tendencia global), los ecosistemas marinos costeros colombianos se vean fuertemente afectados, así como sus bienes y servicios, con el consecuente impacto socioeconómico desastroso para el país.

\section{Reconocimientos y agradecimientos}

El presente trabajo se desarrolló en el marco de la línea de investigación "Dimensión biogeofísica y socioeconómica del cambio global" del Programa de Postgrados de Geografía del Departamento de Geografía, Facultad de Ciencias Humanas, de la Universidad Nacional de Colombia, a cargo del grupo de investigación "Tiempo, clima y sociedad". Agradecemos a la Dirección de Investigación de la Sede Bogotá de la Universidad por su apoyo a través del Quinto Corte, Modalidad 1 (Apoyo a proyectos de tesis y trabajos de investigación), así como al Instituto de Investigaciones Marinas y Costeras "José Benito Vives de Andréis" (Invemar) y al Instituto de Hidrología, Meteorología y Estudios Ambientales (IDEAM), entidades que suministraron los datos necesarios para el desarrollo de la investigación.

\section{Conflicto de intereses}

Los autores declaran que no tienen conflicto de intereses.

\section{Bibliografía}

Alvarado E. M., Duque, F., Flórez, L., Ramírez, R. 1986. Evaluación cualitativa de los arrecifes coralinos de Islas del Rosario (Cartagena-Colombia). Boletín Ecotrópica: Ecosistemas Tropicales. 15: 1-30.

Anlauf, H., D'Croz, L., O'Dea, A. 2011. A corrosive concoction: The combined effects of ocean warming and acidification on the early growth of a stony coral are multiplicative. Journal of Experimental Marine Biology and Ecology. 397: 13-20. 
Anthony, K. R. M., Kline, D. I., Díaz-Pulido, G., Dove, S., Hoegh-Guldberg, O. 2008. Ocean acidification causes bleaching and productivity loss in coral reef builders. Proceedings of the National Academy of Sciences of the United States of America. 105 (45): 17442-17446.

Arnason, R. 2012: Global warming: New challenges for the common fisheries policy? Ocean and Coastal Management, 70: $4-9$.

Bak R.P.M., Nieuwland, G., Meesters, E. H. 2009. Coral growth rates revisited after 31 years: what is causing lower extension rates in Acropora palmata? Bulletin of Marine Science. 84, p. 287-294.

Barange, M., Cheung, W. W. L., Merino, G., Perry, R. I. 2010. Modeling the potential impacts of climate change and human activities on the sustainability of marine resources. Current Opinion in Environmental Sustainability. 2: 326-333.

Barbier, E. B. 2012. A spatial model of coastal ecosystem services. Ecological Economics. 78: 70-79.

Barreto, M. B. 2008. Diagnostics about the state of mangroves in Venezuela: Case studies from The National Park Morrocoy and Wildlife Refuge Cuare. In: Mangroves and Halophytes: Restoration and utilization. p. 51-64.

Brierley, A. S. \& Kingsford, M. J. 2009. Impacts of climate change on marine organisms and ecosystems. Current Biology. 19: R602-R614.

Caldeira, K. \& Wickett, M. E. 2003. Anthropogenic carbon and ocean $\mathrm{pH}$. Nature. 425: 365-365.

Comeaux, R. S., Allison, M. A., Bianchi, T. S. 2012. Mangrove expansion in the Gulf of Mexico with climate change: Implications for wetland health and resistance to rising sea levels. Estuarine, Coastal and Shelf Science. 96: 81-95.

Cooley, S. R., Kite-Powell, H. L., Doney, S. C. 2009. Ocean acidification's potential to alter global marine ecosystem services. Oceanography I. 22 (4): 172-181.

Corporación Colombiana Internacional - CCI. 2006. Pesca y Acuicultura Colombia 2006. Bogotá, D. C., Colombia. 138 p.

Crabbe, M. J. C. 2009. Modelling of geoengineering options in response to climate change and global warming: Implications for coral reefs. Computational Biology and Chemistry. 33: 415-420.

Departamento Nacional de Planeación - DNP. 2007. Visión Colombia II Centenario 2019: Aprovechar el territorio marino-costero en forma eficiente y sostenible. Excelsior Impresores. Bogotá, DC. Colombia. 100 p.

Díaz, J. M., Barrios, L. M., Cendales, M. H., GarzónFerreira, J., Geister, J., López-Victoria, M., Ospina, G. H., Parra-Velandia, F., Pinzón, J., Vargas-ángel, B., Zapata, F. A., Zea, S. 2000. Áreas coralinas de Colombia. Invemar. Serie de Publicaciones Especiales No. 5. Santa Marta, Colombia. 176 p.

Díaz, J. M., Barrios, L. M., Gómez-López, D. I. (Editores). 2003. Las praderas de pastos marinos en Colombia: estructura y distribución de un ecosistema estratégico. Invemar. Serie de Publicaciones Especiales No. 10. Santa Marta, Colombia. 160 p.
Díaz, J. M., Vieira, C. A., Melo, G. J. (Editores). 2011. Diagnóstico de las principales pesquerías del Pacífico colombiano. Fundación Marviva - Colombia. Bogotá, D. C. 242 p.

Dmitrenko, I. A., Polyakov, I. V., Kirillov, S. A. , Timokhov, L. A., Frolov, I. E., Sokolov, V. T., Simmons, H. L., Ivanov, V. V., Walsh, D. 2008. Toward a warmer Arctic Ocean: Spreading of the early $21^{\text {st }}$ century Atlantic Water warm anomaly along the Eurasian Basin margins. Journal of Geophysical Research - Oceans. 113: C05023.

Doney S. C. 2006. The dangers of ocean acidification. Scientific American, March. p. 58-65.

Echavarría-Heras, H. A., Solana-Arellano, E., Franco-Vizcaíno, E. 2006. The role of increased sea surface temperature on eelgrass leaf dynamics: Onset on El Niño as a proxy for global climate change in San Quintín Bay, Baja California. Bulletin Southern California Academy of Sciences. 105 (3): 113-127.

Feely, R. A., Doney, S. C., Cooley, S. R. 2009. Ocean acidification: Present conditions and future changes in a high- $\mathrm{CO}_{2}$ world. Oceanography. 22 (4): 36-47.

Feely, R. A., Alin, S. R., Newton, J., Sabine, C. L., Warner, M., Devol, A., Krembs, C., Maloy, C. 2010. The combined effects of ocean acidification, mixing and respiration on $\mathrm{pH}$ and carbonate saturation in an urbanized estuary. Estuarine, Coastal and Shelf Science. 88: 442-449.

Fischlin, A., Midgley, G. F., Price, J. T., Leemans, R., Gopal, B., Turley, C., Rounsevell, M. D. A., Dube, O. P., Tarazona, J., Velickho, A. A. 2007. Ecosystems, their properties, goods, and services. In: Climate Change 2007: Impacts, Adaptation and Vulnerability. Contribution of Working Group II to the Fourth Assessment Report of the Intergovernmental Panel on Climate Change. Parry, M. L., O. F. Canziani, J. P. Palutikof, P. J. varn del Linden and C. E. Hanson (Eds.). Cambridge University Press. Cambridge, United Kingdom and New York, USA. p. 211-272.

Friedrich, T., Timmermann, A., Abe-Ouchi, A., Bates, N.R., Chikamoto, M.O., Church, M.J., Dore, J.E., Gledhill, D.K., González-Dávila, M., Heinemann, M., Ilyina, T., Jungclaus, J. H., McLeod, E., Mouchet, A., SantanaCasian, J.M. 2012. Detecting regional anthropogenic trends in ocean acidification against natural variability. Nature Climate Change. 20. Publicado en línea: 22 de enero, 2012. Doi: 10.1038/NCLIMATE1372

Fukasawa, M., Freeland, H., Perkin, R., Watanabe, T., Uchida, J., Nishina, A. 2004. Bottom water warming in the North Pacific Ocean. Nature. 427 (6977): 825-827.

Galbraith, P. S., Larouche, P., Chassé, J., Petrie, B. 2012. Seasurface temperature in relation to air temperature in the Gulf of St. Lawrence: Interdecadal variability and long term trends. Deep Sea Research II, 77-80: 10-20.

Garzón-Ferreira, J., Cortés, J., Croquer, A., Guzmán, H., Leao, Z., Rodríguez-Ramírez, A. 2002. Status of coral reefs in Southern Tropical America in 2000-2002: Brazil, Colombia, Costa Rica, Panamá and Venezuela. In: Status of coral reef of the world. Australian Institute of Marine Science. p. 343-360. 
Gledhill, D. K., Wanninkhof, R., Millero, F. J., Eakin, M. 2008. Ocean acidification of the Greater Caribbean Region 1996-2006, J. Geophys. Res. 113: C10031; doi:10.1029/2007JC004629.

Gomes Soares, M. L., Duque-Estrada, G. C., Fernández, V., Pereira-Tognella, M. M. 2012. Southern limit of the Western South Atlantic mangroves: Assessment of the potential effects of global warming from a biogeographical perspective. Estuarine, Coastal and Shelf Science. 101: 44-53.

Grafton, R. Q. 2010. Adaptation to climate change in marine capture fisheries. Marine Policy. 34 (3): 606-615.

Hoegh-Guldberg, O. 2005. Climate change and marine ecosystems. En: Climate change and biodiversity. Lovejoy, T. and L. Hannah, editors. Yale University. United States of America. p. 256-273.

Instituto de Hidrología, Meteorología y Estudios Ambientales, Instituto Geográfico Agustín Codazzi, Instituto de Investigación de Recursos Biológicos Alexander von Humboldt, Instituto de Investigaciones Ambientales del Pacífico Jhon von Neumann, Instituto de Investigaciones Marinas y Costeras José Benito Vives De Andréis, Instituto Amazónico de Investigaciones Científicas. 2007. Ecosistemas continentales, costeros y marinos de Colombia. IDEAM, IGAC, IAvH, INVEMAR, SINCHI, IIAP. Bogotá, D. C, 276 p. + 37 hojas cartográficas.

Idso, C. \& Ferguson, E. H. 2009. Effects of ocean acidification on marine ecosystems. Science and Public Policy Institute. USA. 43 p.

Iguchi, A., Ozaki, S., Nakamura, T., Inoue, M., Tanaka, Y., Suzuki, A., Kawahata, H., Sakai, K. 2012. Effects of acidified seawater on coral calcification and symbiotic algae on the massive coral Porites australiensis. Marine Environmental Research. 73: 32-36.

Instituto de Hidrología, Meteorología y Estudios Ambientales IDEAM. 1998. El medio ambiente en Colombia. Primera edición. OP Gráficas S. A. Bogotá, D. C., Colombia. 495 p.

Instituto de Investigaciones Marinas y Costeras - Invemar. 2009. Lineamientos para un plan de manejo de arrecifes coralinos del Área Marina Protegida Corales del Rosario, San Bernardo e Isla Fuerte en el contexto de cambio climático. Informe técnico final. Proyecto INAP: Diseño e implementación de un programa de adaptación al cambio climático en las áreas insulares del Caribe continental colombiano. Santa Marta, Colombia. 91 p.

Instituto de Investigaciones Marinas y Costeras - Invemar. 2011. Análisis de las condiciones ambientales históricas del Área Marina Protegida Corales del Rosario, San Bernardo e Isla fuerte y sus implicaciones en el mantenimiento de los arrecifes coralinos. Informe técnico final. Proyecto INAP: Diseño e implementación de un programa de adaptación al cambio climático en las áreas insulares del Caribe continental colombiano. Santa Marta, Colombia. 54 p.

Intergovernmental Panel on Climate Change - IPCC. 2000. Special Report on Emissions Scenarios. Edited by N. Nakicenovic and R. Swart. Cambridge University Press. Cambridge, UK and New York, NJ, USA.
Intergovernmental Panel on Climate Change - IPCC. 2013. Climate Change 2013: The Physical Science Basis. Contribution of Working Group I to the Fifth Assessment Report of the Intergovernmental Panel on Climate Change Stocker, T.F., D. Qin, G.-K. Plattner, M. Tignor, S.K. Allen, J. Boschung, A. Nauels, Y. Xia, V. Bex and P.M. Midgley (Eds.). Cambridge University Press, Cambridge, United Kingdom and New York, NY, USA. 1535 p.

Ishimatsu, A., Hayashi, M., Kikkawa, T. 2008. Fishes in high$\mathrm{CO}_{2}$, acidified oceans. In: Effects of ocean acidification on marine ecosystems. Marine Ecology Progress Series. 373: 295-302.

Jackson D. L. \& Wick, G. A. 2010. Near-surface air temperature retrieval derived from AMSU-A and sea surface temperature observations. J. Atmos. Oceanic Techno. 27: 1769-1776.

Jennerjahn, T. C. 2012. Biogeochemical response of tropical coastal systems to present and past environmental change. Earth Science Reviews, 114: 19-41.

Kennedy, V. S., Twilley, R. R., Kleypas, J. A., Cowan Jr., J. M., Hare, S. R. 2002. Coastal and marine ecosystems \& global climate change. PEW Center on Global Climate Change. Arlington, USA. 64 p.

Khatiwala, S., Primeau, F., Hall, T. 2009. Reconstruction of the history anthropogenic $\mathrm{CO}_{2}$ concentration in the ocean. Nature, 462: 346-U110.

Kleypas, J. A. \& Yates, K. K. 2009. Coral reefs and ocean acidification. Oceanography. 22 (4): 108-117.

Kordas, R. L., Harley, C. D. G., O'Connor, M. I. 2011. Community ecology in a warming world; the influence of temperature on interspecific interactions in marine systems. Journal of Experimental Marine Biology and Ecology. 400: $218-226$

Krief, S., Hendy, E. J., Fine, M., Yam, R., Meibom, A., Foster, G. L., Shemesh, A. 2010. Physiological and isotopic responses of scleractinian corals to ocean acidification. Geochimica et Cosmochimica Acta, 74: 4988-5001.

Laurans, Y., Pascal, N., Binet, T., Brander, L., Clua, E., David, G., Rojat, D., Seidl, A. 2013. Economic valuation of ecosystem services from coral reefs in the South Pacific: Taking stock of recent experience. Journal of Environmental Management. 116: 135-144.

Lough, J. M. \&Cooper, T. F. 2011. New insights from coral growth band studies in an era of rapid environmental change. Earth-Science Reviews. 108: 170-184.

Lyman, J. M., Good, S. A., Gouretski, V. V., Ishii, M., Johnson, G. C., Palmer, M. D., Smith, D. G., Willis, J. K. 2010 Robust warming of the global upper ocean. Nature. 465: 334-337.

Madeira, D., Narciso, L., Cabral, H. N., Vinagre, C. 2012. Thermal tolerance and potential impacts of climate change on coastal and estuarine organisms. Journal of Sea Research. 70: 32-41.

Madin, E. M. P., Ban, N. C., Doubleday, Z. A., Holmes, T. H., Pecl, G. T. Smith, F. 2012. Socio-economic and management implications of range-shifting species in marine systems. Global Environmental Change. 22: 137-146. 
Malone, T., Davidson, M., DiGiacomo, P., Gonçalves, E., Knap, T., Muelbert, J., Parslow, J., Sweijd, N., Yanagai, T., Yap, H. 2010. Climate change, sustainable development and coastal ocean. Information needs. Procedia Environmental Sciences. 1: 324-341.

Martínez, M. L., Intralawan, A., Vásquez, G., Pérez-Maqueo, O., Sutton, P., Landgrave, R. 2007. The coasts of our world: Ecological, economic and social importance. Ecological Economics. 63: 254-272.

Marsh G. 2005. Seawater $\mathrm{pH}$ and anthropogenic carbon dioxide. Argonne National Laboratory. University of Chicago. USA. $15 \mathrm{p}$.

McNeil B.I. 2006. Oceanic implications for climate change policy. Environmental Science \& Policy. 9: 595-606.

Miller, K., Charles, A. C., Barange, M., Brander, K., Gallucci, V. F., Gasalla, M. A., Khan, A., Munro, G., Murtugudde, R., Ommer, R. E., Perry, R. I. 2010. Climate change, uncertainty, and resilient fisheries: Institutional responses through integrative science. Progress in Oceanography. 87 (1-4): 338-346

Mooney, H., Larigauderie, A., Cesario, M., Elmquist, T., HoeghGuldberg, O., Lavorel, S., Mace, G. M., Palmer, M., Scholes, R., Yahara, T. 2009. Biodiversity, climate change and ecosystem services. Current Opinion in Environmental Sustainability. 1: 46-54.

Nakano, Y. \& Watanabe, Y. W. 2005. Reconstruction of pH in the surface seawater over the North Pacific Basin for all seasons using temperature and chlorophyll- $a$. Journal of Oceanography. 61: 673-680.

Pabón, J. D. 2012. Cambio climático en Colombia: tendencias en la segunda mitad del siglo XX y escenarios posibles para el siglo XXI. Rev. Acad. Colomb. Cienc. 36 (139): 127-144.

Perry, R. I., Barange, M., Ommer, R. E. 2010a. Global changes in marine systems: A social-ecological approach. Progress in Oceanography. 87 (1-4): 331-337.

Perry, R. I., Ommer, R. E., Barange, M., Werrner, F. 2010b. The challenge of adapting marine social-ecological systems to the additional stress of climate change. Current Opinion in Environmental Sustainability, 2: 356-363.

Pierce, D. W., Barnett, T. P., AchutaRao, K. M., Glecker, P. J., Gregory, J. M., Washington, W. M. 2006. Anthropogenic warming of the ocean: Observations and model results. Journal of Climate. 19: 1873-1900.

Porzio, L., Buia, M. C., Hall-Spencer, J. M. 2011. Effects of ocean acidification on macroalgal communities. Journal of Experimental Marine Biology and Ecology. 400 (1-2): 278-287.

Putnam, H. M. \& Edmunds, P. J. 2011. The physiological response of reef corals to diel fluctuations in seawater temperature. Journal of Experimental Marine Biology and Ecology. 396 (2): 216-223.

Remoundou, K., Koundouri, P., Kontogianni, A., Nunes, P. A. L. D., Skourtos, M. 2009. Valuation of natural marine ecosystems: An economic perspective. Environmental Science \& Policy. 12: 1040-1051.
Rojas, P. J. 2013. La expresión del cambio global en las variables físico-químicas del medio marino regional y su impacto en los ecosistemas marino-costeros colombianos. Tesis para optar al título de Magister en Geografía. Universidad Nacional de Colombia. Bogotá, D. C., Colombia. 118 p.

Rodrigues, L. C., van den Bergh, J. C. J. M., Ghermandi, A. 2013. Socio-economic impacts of ocean acidification in the Mediterranean Sea. Marine Policy. 38: 447-456.

Ruckelshaus, M., Doney, S. C., Galindo, H. M., Barry, J. P., Chan, F., Duffy, J. E., English, C. A., Gaines, S. D., Grebmeier, J. M., Hollowed, A. B., Knowlton, N., Polovina, J., Rabalais, N. N., Sydeman, W. J., Talley, L. D. 2013. Securing ocean benefits for society in the face of climate change. Marine Policy. 40: 154-159.

Ruiz, F. 2010. Cambio climático en temperatura, precipitación y humedad relativa para Colombia usando modelos meteorológicos de alta resolución (panorama 2011-2100). Nota Técnica de IDEAM, No. IDEAM-METEO/005-2010, Bogotá D.C., 91 p.

Sheppard, C. \&Rioja-Nieto, R. 2005. Sea surface temperature 1871-2099 in 38 cells in the Caribbean region. Marine Environmental Research. 60 (3): 389-396.

Short, F. T. \& Neckles, H. A. 1999. The effects of global climate change on seagrasses. Aquatic Botany. 63 (3-4): 169-196.

Solano, O. D. 1994. Corales, formaciones arrecifales y blanqueamiento de 1987 en Bahía Portete (Guajira, Colombia). An. Inst Invest. Mar. Punta Betín. 23: 149-163.

The Royal Society. 2005. Ocean acidification due to increasing atmospheric carbon dioxide. Policy document. The Clyvedon Press Ltd. Cardiff, UK. 60 p.

Vargas-Ángel, B., Zapata, F. A., Hernández, H., Jiménez, J. M. 2001. Coral and coral responses to the 1997-98 El Niño event on the Pacific coast of Colombia. Bulletin of Marine Science. 69 (1): 111-132.

Veron, J. E. N., Hoegh-Guldberg, O., Lenton, T. M., Lough, J. M., Obura, D. O. , Pearce-Kelly, P., Sheppard, C. R. C., Spalding, M. , Stafford-Smith M. G., Rogers, A. D. 2009. The coral reef crisis: The critical importance of $<350$ ppm $\mathrm{CO}_{2}$. Marine Pollution Bulletin. 58: 14281436 .

Waycott, M., Collier, C., McMahon, K., McKenzie, R. P., Udy, L., Alana, J., Alana, G. 2007. Vulnerability of seagrasses in the Great Barrier Reef to climate change. In: Climate Change and the Great Barrier Reef: A vulnerability assessment. Great Barrier Reef Marine Park Authority and Australian Greenhouse Office. Townsville, QLD, Australia. p. 193-236.

Widdicombe, S. \& J. I. Spicer. 2008. Predicting the impact of ocean acidification on benthic biodiversity: What can animal physiology tell us? Journal of Experimental Marine Biology and Ecology. 366: 187-197.

Zapata, L. A. 2002. Effects of El Niño and La Niña on the Fisheries of the Colombian Pacific. Investig. Mar. 30 (1): 205-206. 